\title{
On the Modeling and Simulation of Variable-Length Pendulum Systems: A Review
}

\author{
Godiya Yakubu $^{1}$ (D $\cdot$ Paweł Olejnik $^{1} \cdot$ Jan Awrejcewicz ${ }^{1}$
}

Received: 19 April 2021 / Accepted: 30 September 2021 / Published online: 2 March 2022

(c) The Author(s) 2022

\begin{abstract}
A comprehensive review of variable-length pendulums is presented. An attempt at a unique evaluation of current trends in this field is carried out in accordance with mathematical modeling, dynamical analysis, and original computer simulations. Perspectives of future trends are also noted on the basis of various concepts and possible theoretical and engineering applications. Some important physical concepts are verified using dedicated numerical procedures and assessed based on dynamical analysis. At the end of the review, it is concluded that many variable-length pendulums are very demanding in the modeling and analysis of parametric dynamical systems, but basic knowledge about constant-length pendulums can be used as a good starting point in providing much accurate mathematical description of physical processes. Finally, an extended model for a variable-length pendulum's mechanical application being derived from the Swinging Atwood Machine is proposed. The extended SAM presents a novel SAM concept being derived from a variable-length double pendulum with a suspension between the two pendulums. The results of original numerical simulations show that the extended SAM's nonlinear dynamics presented in the current work can be thoroughly studied, and more modifications can be achieved. The new technique can reduce residual vibrations through damping when the desired level of the crane is reached. It can also be applied in simple mechatronic and robotic systems.
\end{abstract}

\section{List of Symbols}

$\omega$

$\beta$

$u$

$a$

sion point of the pendulum, $\mathrm{m}$

$a_{0} \quad$ Portable acceleration, $\mathrm{m} / \mathrm{s}^{2}$

$q \quad$ Model respond parameter

$\omega_{0} \quad$ Mean resonance frequency, $\mathrm{Hz}$

$b_{0} \quad$ Time-average oscillator damping

$E(t) \quad$ External driving force, $\mathrm{N}$

$h_{0} \quad$ Damping variable in the damping and resonant frequency

$f(t) \quad$ Mathieu/periodic function with minimal period $\pi$

$r \quad$ Amplitude of excitation, $\mathrm{m}$

Godiya Yakubu

godiya.yakubu@dokt.p.lodz.pl

1 Department of Automation, Biomechanics and Mechatronics, Faculty of Mechanical Engineering, Lodz University of Technology, 1/15 Stefanowski Str., 90-924 Lodz, Poland
Angular displacement, rad

Angular velocity, $\mathrm{rad} / \mathrm{s}$

Angular acceleration, $\mathrm{rad} / \mathrm{s}^{2}$

$l \quad$ Length of the pendulum, $\mathrm{m}$

$l_{0} \quad$ Average value of the pendulum length, $\mathrm{m}$

$l(t) \quad$ Pendulum displacement, $\mathrm{m}$

$\dot{l}(t) \quad$ Pendulum velocity, $\mathrm{m} / \mathrm{s}$

$\ddot{l}(t) \quad$ Pendulum acceleration, $\mathrm{m} / \mathrm{s}^{2}$

$\mu \quad$ Gain coefficient of exponential amplitude

$h \quad$ Depth amplitude modulation, $\mathrm{m}$

$a(t), b(t) \quad$ Varying envelope function

$m \quad$ Mass of the pendulum, $\mathrm{kg}$

$F \quad$ Control action force

$\xi(t) \quad$ Harmonic oscillator with zero-average perturbation time-dependent

$\gamma, \lambda \quad$ Parameters of the oscillatory system

$z \quad$ Generic time-dependent function

A Amplitudes of the periodic forcing, $\mathrm{m}$

$A_{i} \quad$ Arbitrary amplitudes, $\mathrm{m}$

$\omega_{i} \quad$ Excitation frequencies, $\mathrm{rad} / \mathrm{s}$

$\phi_{i} \quad$ Phase shifts, rad

$\dot{x} \quad$ Velocity $\mathrm{m} / \mathrm{s}$

$\ddot{x} \quad$ Acceleration $\mathrm{m} / \mathrm{s}^{2}$

$f_{0} \quad$ Excitation force, $\mathrm{N}$ 


$\begin{array}{ll}T & \text { Kinetic energy, Nm } \\ T_{s} & \text { Sum of kinetic energy of a slider, Nm } \\ T_{b} & \text { Sum of kinetic energy for a pendulum, Nm } \\ U & \text { Potential energy, Nm } \\ c & \text { Viscous damping coefficient, Ns/m } \\ k & \text { Spring constant, N/m } \\ M & \text { Counter mass/travelling and hoisting compo- } \\ & \text { nent, kg } \\ \theta & \text { Angular position of flywheel, rad } \\ T_{f} & \text { Frictional torque, Nm } \\ x & \text { Horizontal displacement/slider displacement, } \mathrm{m} \\ \varepsilon & \text { Forcing or pertubation parameter } \\ y & \text { Vertical displacement, } \mathrm{m} \\ y_{m} & \text { Mass vertical displacement, } \mathrm{m} \\ y_{e} & \text { Beam elastic displacement in } y \text {-direction, } \mathrm{m} \\ x_{e} & \text { Beam elastic displacement in } x \text {-direction, } \mathrm{m} \\ v & \text { Relative speed/velocity, } \mathrm{m} / \mathrm{s} \\ I & \text { Moment of the inertia, kgm }{ }^{2} \\ I_{c} & \text { Constant of inertial of all part, } \mathrm{kgm}^{2} \\ I_{v} & \text { Adjustable inertia, kgm }{ }^{2} \\ \mu_{f} & \text { Coefficient of friction } \\ \mu_{m} & \text { Mass ratio } \\ R & \text { Radius, } \mathrm{m} \\ \Delta & \text { Difference }\end{array}$

\section{Introduction}

The variable-length pendulum may be treated as a secondorder nonlinear differential equation with a step function dependent coefficients which can be transformed into equivalent discrete dynamical systems $[1,2]$. It can also be treated as a control system due to time-varying control laws of changing its length [3], as well as it has various applications in mechatronic systems, which include: robots, electromechanical systems like induction motors, purely electrical networks like dc-dc power converters, lifting devices like mine elevators or cranes, earthquakes detection based on various concepts of inverted pendulums or even wave energy converters (WEC) $[4,5]$.

We begin from some methods of forcing of the analyzed dynamical system. Work [6] proves the effectiveness of real experimental data of reconfiguration of coupled pendulums on a data flow visual programming using LabVIEW. The Lyapunov exponents and forcing amplitude in controlling the chaotic motion of a driven pendulum is studied in [7]. [8] applied the Poincaré-Birkhoff fixed point theorem and shows that there exist a stable and unstable periodic solution of a forced pendulum of variable length. [9-12] indicate that if a threshold velocity is adequately chosen to regulate a rotational pendulum's control action, the controlled pendulum can reach stable rotations for both forcing scenarios, irrespective of the initial conditions and forcing parameters.
The variable-length pendulum is a physical concept associated with parametric oscillations governed by certain forms of differential equations and functional principles. A parametric oscillator can be treated as a harmonic oscillator whose physical features change over time [13]. Some specific time-dependent variables are associated with the resonance frequency or damping of the oscillator.

The most known oscillator is given as a linear secondorder differential equation in the form:

$\frac{d^{2} x(t)}{d t^{2}}+\beta(t) \frac{d x(t)}{d t}+\omega^{2}(t) x(t)=0$

where $\omega$-the time-dependent parameter changing with the period $T, \beta$-constant. For instance, coefficient of the parametric oscillator can be identified with the use of semi-empirical methods based on a coupled oscillator approach [14].

In [15], the fundamental behavior of parametrically excited Kochin oscillator model with double degeneracy of frequencies was referenced to Mathieu's oscillator. It follows that there is a structural instability of parametric oscillations. [16] finds out that thwarting modifies the dynamics of two and three coupled parametric oscillators after being theoretically analyzed using a nonlinear Mathieu equation and validated by numerical simulations. The complexity and asymmetry of periodic motions of a parametric Duffing oscillator are dependent on the contribution of harmonic amplitudes of the periodic signal $[17,18]$. The oscillating pendulum amplitude can be suppressed efficiently by a controllable moving mass [19-21].

Parametric resonance takes place in dynamical processes when the external frequency is twice the system's natural frequency as in the case of the vertically forced pendulum and the result is given by the Mathieu equation [22]:

$\ddot{u}(t)+(a+\beta \cos t) u(t)=0$,

where $u(t)$-perturbation from the periodic equation, $\beta \cos t$ -an energy source of parametric excitation [13].

Mathieu functions sometimes called angular Mathieu functions are the solutions of Mathieu's differential equation given in the form:

$\frac{d^{2} y(t)}{d t^{2}}+(a-2 q \cos 2 x(t)) y(t)=0$,

where $a, q$-model parameters, $x(t)$ is the linear term, i.e. Eq. (3) is linear with respect to $x(t)$ [13]. The equation of the parametric oscillator in Eq. (1) can be extended to a non-homogeneous one by adding an external driving force $\mathrm{E}(t)$, governed by:

$\frac{d^{2} x(t)}{d t^{2}}+\beta(t) \frac{d x(t)}{d t}+\omega^{2}(t) x(t)=\mathrm{E}(t)$. 
Considering the external driving force to be the mean resonance frequency $\omega_{0}$, that is $\mathrm{E}(t)=\mathrm{E}_{0} \sin \omega_{0} t$, the solution to Eq. (4) is given as follows:

$x(t)=\frac{2 \mathrm{E}_{0}}{\omega_{0}^{2}\left(2 \mathrm{~b}_{0}-\mathrm{h}_{0}\right)} \cos \omega_{0} t$.

A parametric oscillator is a harmonic oscillator with timevarying physical properties. Parameters $\omega_{0}$, and $\beta$, are timedependent. Therefore, it is assumed to vary periodically with the same period $T$. In finding the solution of Eq. (1) we assumed the damping to be sufficiently strong so that the amplitude of the parametric oscillations in the absence of the driving force $E$ does not diverge. At this state, the parametric pumping slip to lower the effective damping in the system. If the solution in Eq. (5) will be dependent on constant $b_{0}$, and $\mathrm{h}_{0}$, then as $\mathrm{h}_{0}$, approaches the threshold, $2 \mathrm{~b}_{0}$, the amplitude diverges. When $h_{0}$ is greater than or equal to $2 b_{0}$, the system enters a parametric resonance, and the amplitude begins to increase exponentially even in the absence of a driving force $\mathrm{E}(t)$.

When the Mathieu function $f(t)$, varies periodically, then one finds a particular as an example of Hill equation [23], i.e. Mathieu equation when $f(t)$ is a simple sine wave, i.e.:

$\frac{d^{2} y(t)}{d t^{2}}+f(t) y(t)=0$

where $f(t)$ has the minimal period $\pi$, that is $f(t+\pi)=f(t)$.

The special case of the Hill equation presented with the periodic function is called the Meissner equation [24, 25], of which example can be written in many different ways, and for instance:

$\frac{d^{2} y(t)}{d t^{2}}+\left(1+r \frac{\sin (\omega t)}{|\sin (\omega t)|}\right) y(t)=0$.

Many authors and researchers have applied various methods for analyzing dynamical systems (variable-length or parametric pendulums). Some of the system equations studied by many authors and their main conclusions are summarized in the next Section.

\section{The Variable-Length Pendulum Systems}

In [26], the authors provide the equation of motion of a simple pendulum with a fixed length $l_{0}$ as:

$\ddot{\varphi}(t)+\frac{g}{l_{0}} \sin \varphi(t)=0$,

$\varphi$-angle between the pendulum and the downward vertical axis, $g$-acceleration due to gravity, and dots denote the second derivative with respect to time $t, l_{0}$-constant length of the pendulum.

For the linearized system:

$\ddot{\varphi}(t) \pm(\alpha-\delta \cos \tau) \varphi(t)+b \dot{\varphi}(t)=0$,

where $\alpha=\frac{g}{l_{0} \omega^{2}}, \delta=\frac{g}{l_{0}}, \tau=\omega t, \omega$-natural frequency of oscillation of the system, $b$-damping coefficient, and $\delta$ is equivalent to the $\pi$ phase shift in time $t . \pm$ is used to present two forms of differential equations in the literature. Taking into account the above, Hill equation is obtained:

$\ddot{\varphi}(t)+\frac{\alpha+\varepsilon \cos t}{1+\varepsilon \cos t} \varphi(t)=0$,

where $\varepsilon=l_{1} / l_{0}<1, l(t)=l_{0}+l_{1}$-length with periodic variation with respect to time $t$. Parameter $\delta$ and $\varepsilon$ are the perturbation parameters.

Successively, the Mathieu equation was found by keeping the terms in Eq. (10) at first order in $\varepsilon$

$\ddot{\varphi}(t)+(\alpha+\varepsilon(1-\alpha) \cos t) \varphi(t)=0$.

The bifurcation effect of the oscillation is based on the Mathieu equation. Its relates to a period-doubling bifurcation, which in turn leads to chaos due to the period-doubling cascade. It was observed that decreasing the damping coefficient $b$ leads to a decrease in the periodic attractors of the basin of attraction. A pendulum with constant length can be stabilized around the upward position for any parameter values, which is different from a pendulum with a varying length that can not be stabilized around the upward position for any parameter values.

Work [27] demonstrates the equation of motion for a laboratory parametric oscillator as presented in Fig. 1, i.e.:

$\ddot{\varphi}(t)+2 \frac{\dot{l}(t)}{l(t)} \dot{\varphi}(t)+2 b \dot{\varphi}(t)+\frac{g}{l(t)} \varphi(t)=0$,

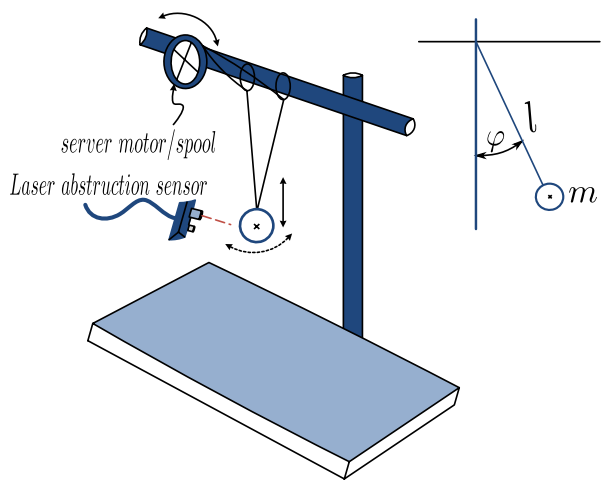

Fig. 1 Schematic of the parametrically driven pendulum 
where $2 \frac{i(t)}{l(t)}$ is the parametric driving. The solution is found in Eqs. $(14,15)$ in the form of a coefficient for cosine and sine functions, respectively of Eq. (13).

$$
\begin{aligned}
& \varphi(t)=a(t) \sin \left(\omega_{0} t\right)+b(t) \sin \left(\omega_{0} t\right), \\
& a(t)\left(\frac{3}{2} h \omega_{0}^{2}+2 \mu \omega_{0}+2 b \omega_{0}+2 b \mu h\right) \\
& \quad+b(t)\left(2 b \mu+2 h \mu \omega_{0}-h \mu \omega_{0}-h b \omega_{0}\right)=0 \\
& a(t)\left(2 b \mu-2 h \mu \omega_{0}+h \mu \omega_{0}+h b \omega_{0}\right) \\
& \quad+b(t)\left(\frac{3}{2} h \omega_{0}^{2}-2 \mu \omega_{0}-2 b \omega_{0}-b \mu h\right)=0,
\end{aligned}
$$

$\mu$-gain coefficient of exponential amplitude, $h$-depth of modulation of the amplitude, $a(t), b(t)$-varying envelope functions.

The system's mechanical strength is maintained by increasing or decreasing the amount of modulation through the parametric resonance. The experiment demonstrates chaotic properties of the system that allow for observation of nonlinear dynamics behaviour.

In [28], the authors solve a problem of damped sway of a suspended payload that was achieved by moving a pivot base in vertical direction, as it can be seen in Figs. 2, 3, 4.

The second-order nonlinear dynamical system is governed by the balancing momentum equation:

$y(t)=y_{0}+\Delta y(t) \sin (2 \omega t)$,

$$
\begin{gathered}
\left(m_{1}+m_{2}\right) \ddot{y}(t)+b \dot{y}(t)=F(t)-\left(m_{1}+m_{2}\right) g \\
-m_{2} l\left(\ddot{\varphi}(t) \sin \varphi(t)+\dot{\varphi}(t)^{2} \cos \varphi(t)\right),
\end{gathered}
$$

where $m_{1}$-a point-focused forced mass of the mathematical pendulum of length $l ; m_{2}$-the second mass at the end of

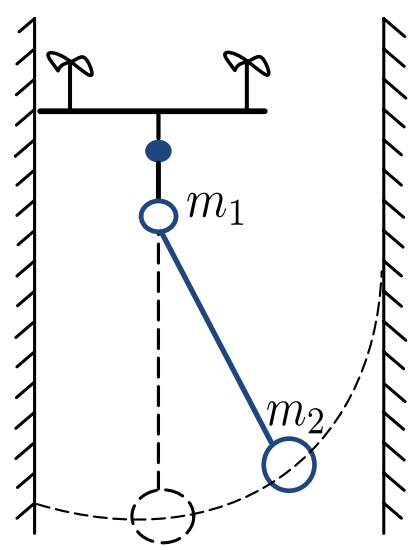

Fig. 2 Problem motivation and formulation of the constrained space for a quadrocopter with suspended load

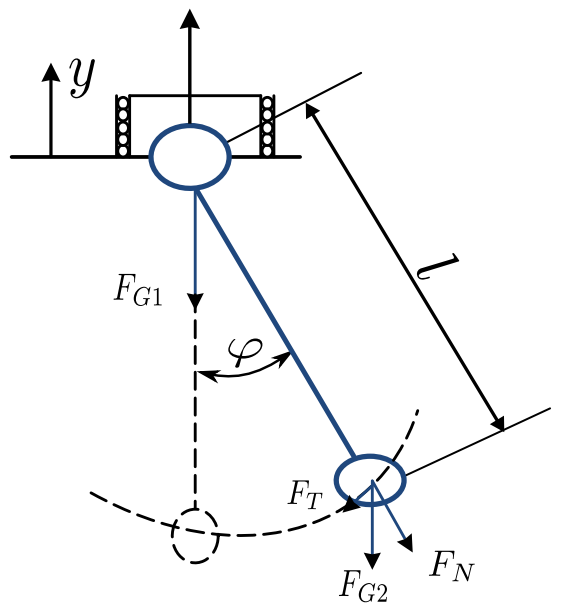

Fig. 3 Problem motivation and formulation of the simplified model of the pendulum with moving base

the pendulum; $F$-the control action force that also keeps the system from free-falling by preliminary compensating the gravity force.

The experimental validation is done by considering the horizontal motion only. The simulation validation is done on a nonlinear two-dimensional model of a quadrocopter carrying a suspended payload using the Lyapunov approach's nonlinear control feedback design. The proposed system finds its application in a crane, where it can be used to damp the residual vibrations when the desired crane position is reached. Other areas of applications can be found in mechatronic systems and flexible robotics-a pendulum-like robot [29-31], where similar problems can occur.

In [32], the comparative Fourier and wavelet approach is used to analyze the motion of a variable-length pendulum. The equation of motion is presented as follows:

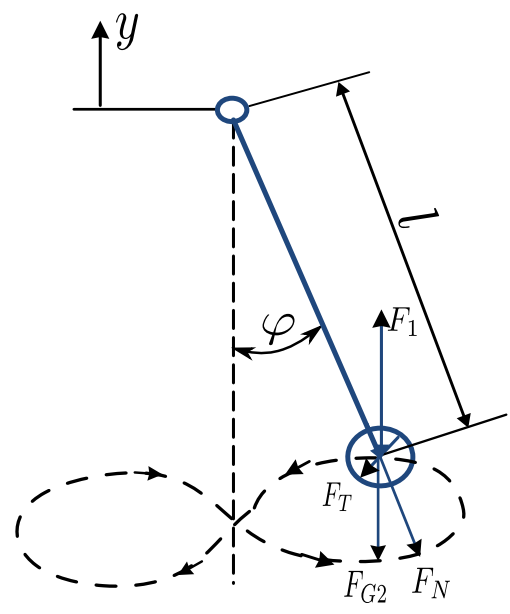

Fig. 4 Problem motivation and formulation of the further simplified model to a pendulum with a free moving mass pivot 
$m \ddot{s}(t)=-m g \sin \varphi(t)$,

where $s(t)=l(t) \varphi(t)$ is the length spanned by the oscillation during its motion. Finally, the solution is found:

$\varphi(t)=\varphi_{0} \sin (\omega(t) t+\phi)$,

where $\phi$-phase shift, and $\omega(t)=\sqrt{\frac{g}{l(t)}}$.

The oscillation and the length change time intervals indicate a frame for comparison of the signal frequency. Moreover, the wavelength change of the pendulum translation performs better than Fourier transformation did. The two approaches provided an easy-to-interpret visual inspection of time-frequency analysis.

Work [33] describes some aspects of Mathieu and Hill equations with a nonlinear equation and used general asymptotic methods to obtain results, as well as using Matlab to verify the idea. Hill's model is presented with a kinematic simulation of a simple harmonic oscillator with zero-averaged perturbation time-dependent $\xi(\mathrm{t})$ as:

$\ddot{x}(t)+\omega_{0}^{2}[1+\xi(\mathrm{t})] x(t)=0$,

where $x$-displacement, $\omega_{0}$-natural mean resonance frequency of the oscillator.

The Mathieu equation is given in the form:

$\ddot{x}(t)+a(t) \dot{x}+q(t) x(t)=0$,

where, $a(t)$ and $q(t)$ are continuous and have a common period $T$.

The equation of the unstable mode of oscillation system with its phase portrait is given as follows:

$\ddot{x}(t)+\omega_{0}^{2}[1+\sin \varphi(t)] x(t)=0$.

Numerical solution of Eq. (22) is computed and presented in Figs. 5, 6 for the value of $\omega_{0}=20$ and $\varphi=\frac{\omega_{0}}{4}$. The

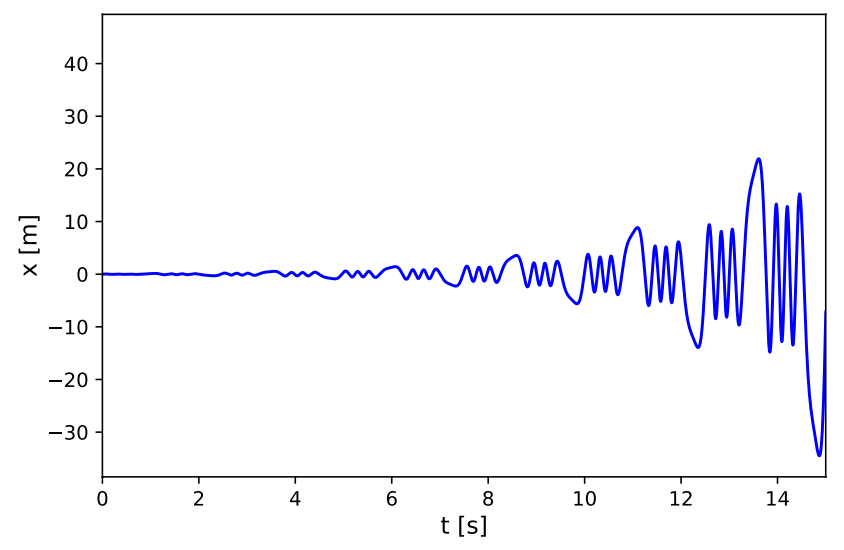

Fig. 5 Unstable time trajectory of the oscillation system in Eq. (22) for $\omega_{0}=20$ and $\varphi=\frac{\omega_{0}}{4}$ asymptotic effect is confirmed in our work using the numerical solution of Eqs. $(23,24)$, as it is presented in Figs. 7, 8, 9, 10 .

$\omega=\sqrt{\left(\omega_{0}^{2}-\gamma^{2}+\varepsilon \lambda \frac{\omega_{0}^{2}}{2}\right)}$,

and

$\ddot{x}(t)+2 \gamma \dot{x}(t)+\omega_{0}^{2}[1+\lambda \sin \beta \mathrm{t}] x(t)=0$,

where $\gamma$ and $\lambda$ are parameters of the oscillatory system, $\omega$ -parametric harmonic excitation frequency, and $\varepsilon$-forcing or perturbation parameter that may cause chaotic dynamics.

In [34] the authors analyze oscillations of a parametric pendulum providing the governed equation of the motion not in the vicinity of equilibrium, as it follows:

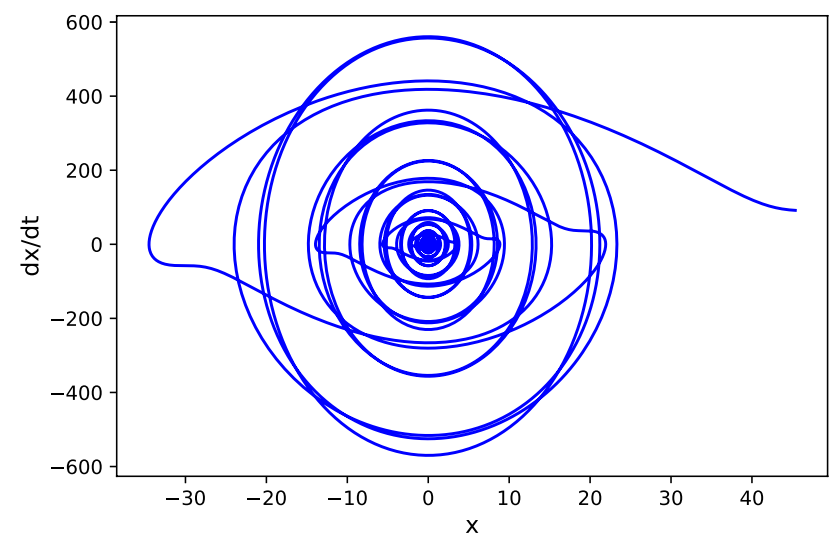

Fig. 6 Phase portrait of the unstable mode of the oscillation system in Eq. (22) for $\omega_{0}=20$ and $\varphi=\frac{\omega_{0}}{4}$

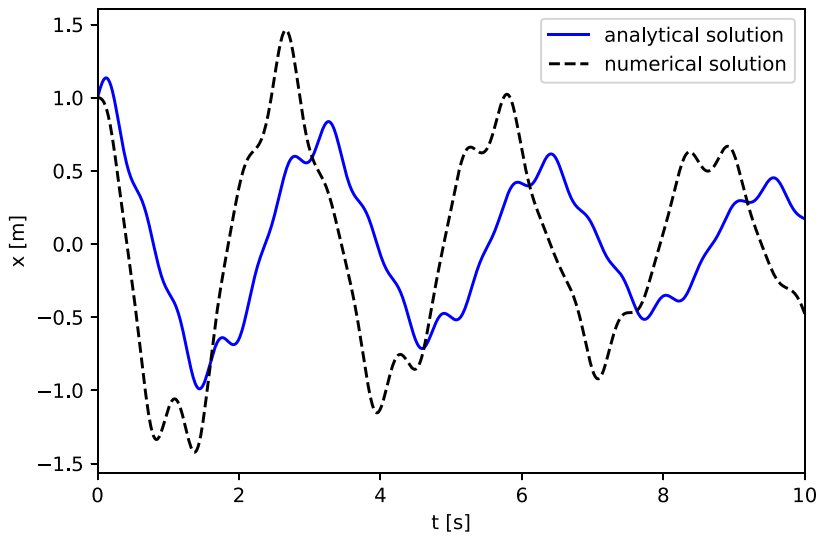

Fig. 7 Comparison between numerical and analytical solution in Eqs. (23) and (24) for the system parameters: $\gamma=0.1, \omega_{0}=\sqrt{2}, \lambda=10$, $\beta=10, \varepsilon=0.196$ 


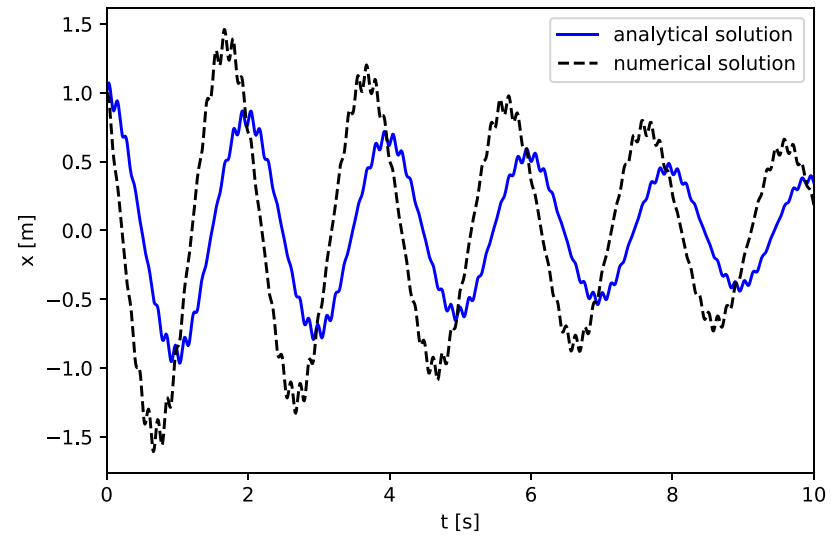

Fig. 8 Comparison between numerical and analytical solution in Eqs. (23) and (24) for the system parameters: $\gamma=0.1, \omega_{0}=\sqrt{2}$, $\lambda=100, \beta=50, \varepsilon=0.08$

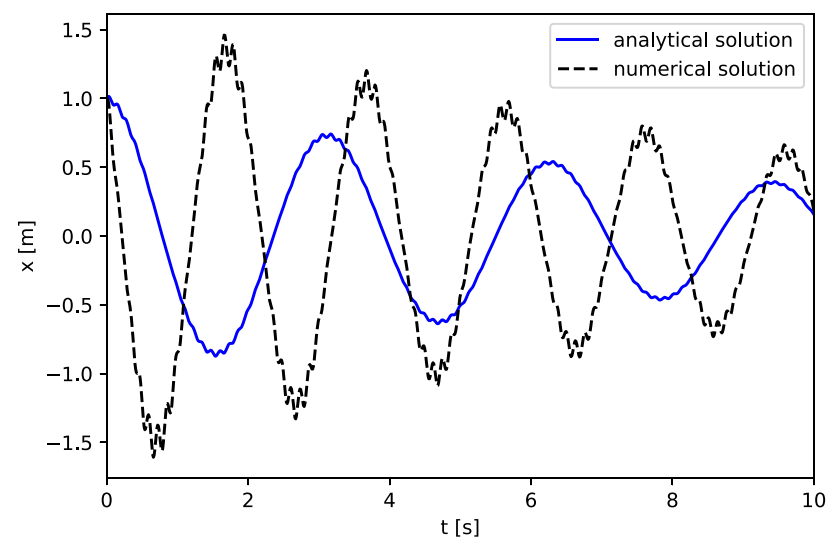

Fig. 9 Comparison between numerical and analytical solution in Eqs. (23) and (24) for the system parameters: $\gamma=0.1, \omega_{0}=\sqrt{2}$, $\lambda=100, \beta=50, \varepsilon=0.02$

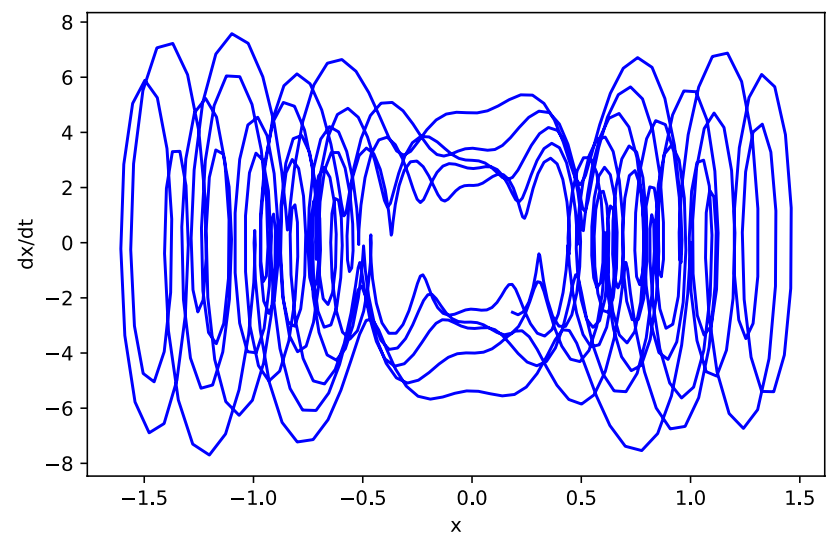

Fig. 10 A stable time trajectory of oscillations on the phase portrait in Eqs. (23) and (24) for the system parameters: $\gamma=0.1, \omega_{0}=\sqrt{2}$, $\lambda=10, \beta=10, \varepsilon=0.196$ $\frac{d^{2} \varphi}{d t^{2}}+\sin \varphi+\varepsilon A \cos ((\omega+\varepsilon s) t) \sin \varphi=0$.

If resonance appears at frequency $\frac{\omega}{2}$ then it follows that

$$
\begin{aligned}
& \frac{d^{2} \varphi}{d t^{2}}+\frac{\omega}{2} \varphi+\varepsilon \mu\left(-\frac{\omega}{2} \varphi+\varphi\right) \\
& \quad+\varepsilon A \cos ((\omega+\varepsilon s) t) \sin \varphi=0 .
\end{aligned}
$$

For $\varepsilon \ll 1, \omega=\frac{\Omega}{\omega_{0}}$ ( $\Omega$-unknown constant), $\mu=\frac{1}{\varepsilon}$, when $\omega$ is depleted from resonance, then complex variables are introduced

$\psi=\left(\dot{\varphi}+i \frac{\omega}{2} \varphi\right), \psi_{j}^{*}=\left(\dot{\varphi}-i \frac{\omega}{2} \varphi\right)$.

where $\varepsilon A$ states the amplitude of the periodic forcing.

The Hamiltonian equation is obtained as below:

$$
\begin{aligned}
H=- & a^{2}\left(\frac{s}{2}+\mu \frac{\omega}{4}\right)-\omega J_{0}\left(\frac{2}{\omega} a\right) \\
& -A \omega J_{2}\left(\frac{2}{\omega} a\right) \cos 2 \delta .
\end{aligned}
$$

The phase planes are investigated, making it possible to predict strongly modulated regimes and large amplitude with initial excitation. The resonance problem can be eliminated by changing the forcing term, i.e. for $t=0$.

Moreover, the work [34] introduces the motion on the complex path and the oscillation effect by specifying the nonlinear dynamics of a pendulum that exaggerates the static and non-stationary oscillations dynamics. The semi-inverse path has been found to represent the pendulum with high powers throughout the semi-linear process. The techniques predict evolution with a weak flexibility.

In [35] we find the equation of a vertical excitation of a pendulum with mass $m$ as it follows:

$$
\begin{aligned}
& L(\varphi, \dot{\varphi}, z, \dot{z})=\frac{1}{2} M l^{2}\left(\dot{\varphi}(t)^{2}+\frac{1}{l^{2}} \dot{z}^{2}(t)\right) \\
& \quad-M l \dot{z}(t) \dot{\varphi}(t) \sin \varphi(t)-M g z(t)-M g l \cos \varphi(t) .
\end{aligned}
$$

Taking $z$ in upright direction, $\mathbf{a}=-g \hat{z}$-gravitational acceleration, $l$-length of pendulum, the equation becomes:

$\frac{d^{2} \varphi(t)}{d t^{2}}=\frac{g}{l}\left(1+\frac{1}{g} \ddot{z}(t)\right) \sin \varphi(t)$.

Taking $\ddot{z}$ to be generic time-dependent function and given approximation for small angles $\sin \varphi=\varphi$ :

$\ddot{\varphi}(t)=\left(\omega^{2}+\frac{1}{l} \ddot{z}(t)\right) \varphi(t)$.

The parametric excitation is considered and simplified to a real inverted pendulum problem, leading to 
$z(t)=\sum_{i=1}^{N} A_{i} \sin \left(\omega_{i} t+\phi_{i}\right)$

where $A_{i}$-arbitrary amplitudes, $\omega_{i}$-excitation frequencies, $\phi_{i}$ -phase shifts, $i=1, \ldots N$.

Considering the more general equation

$\ddot{\varphi}(t)=\left(\omega_{0}^{2}-f(t)\right) \varphi(t)$,

where $\omega_{0}^{2}=1$ and $A_{i}^{*}=A$ results in the following transformation

$f(t)=\frac{1}{l} \sum_{i=1}^{N} A_{i} \omega_{i}^{2} \sin \left(\omega_{i} t+\phi_{i}\right)$.

The applied perturbative analysis and numerical integration explored an inverted pendulum's stability with generating parametric excitation described by a superposition of different sines values. Different frequencies and phase and analytical, critical amplitude for the stabilization has abstained. It is determined that the amplitude depends on the size and mass used as panels for the scale screening units and is tested with different sine values.

The above reviews give a generalized analytical idea on a pendulum with fixed- and variable-length with their possible area of applications. Now, we will look at the variablelength pendulum with stiffness and damping as investigated by different authors. In [36] one finds an analysis without the damping force, while [37-39], and [40] include damping force in the analysis.

\subsection{Variable-Length Pendulum with Stiffness and Damping}

In [36] the equation $y=a \sin \omega t$ for modeling a pendulum harmonic oscillations (as shown in Fig. 11) is considered. It presents the equation for the oscillations of the pendulum as it follows:

$\frac{d}{d t}\left(l^{2}(t) \frac{d \varphi(t)}{d t}\right)=l(t)\left(g+a_{0}\right) \sin \varphi(t)$,

where $\varphi$ is the angular position.

Fig. 11 A variable-length pendulum

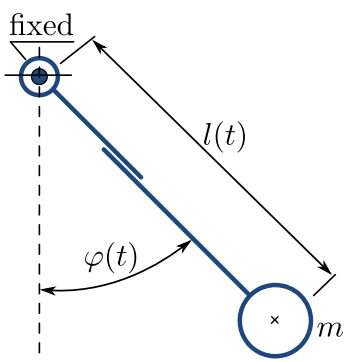

The pendulum's harmonic oscillations depend on periodic variations of the pendulum's length. The largest Lyapunov exponent, bifurcation diagrams and Poincaré maps on phase plane diagrams were shown and inspected. Maps of dynamic modes were obtained, identifying a range of parameters containing 2-periodic cycles, leading to the occurrence of chaos. Thus, a conventional system of a pendulum with a periodically varying length on a vibrating base near the resonance between the oscillation frequencies of the suspension point's length and oscillations has a chaotic behavior in the vicinity of the upper equilibrium position.

In [37], the authors use a semi-analytical method to study the motion of period- 1 to period- 2 bifurcation trees in a periodically forced nonlinear spring pendulum as shown in Fig. 12.

The equation of dynamics describes the concept of such a system as it follows:

$$
\begin{aligned}
& m \ddot{x}(t)-m(l(t)+x) \dot{\varphi}(t)^{2}=m g \cos \varphi(t) \\
& \quad-k_{1} x-k_{2} x^{3}-b \dot{x}(t)-Q_{0} \cos \omega t \sin \varphi(t),
\end{aligned}
$$

and

$$
\begin{aligned}
& m(l+x)^{2} \ddot{\varphi}(t)+2 \dot{x}(t)(l+x) m \dot{\varphi}(t)=-m g(l+x) \\
& \quad \cdot \sin \varphi(t)-b \dot{\varphi}(t)(l+x)^{2}-Q_{0}(l+x) \cos \omega t \cos \varphi(t) .
\end{aligned}
$$

The discrete periodic nodes of periodic motions, the numerical simulations, and the corresponding harmonic amplitude spectrum and phases were presented. Taylor series expansion and perturbation methods were used for the research studies. It was determined that the pendulum spring system dynamics were too large and too complex to be analysed. The spring pendulum system needs to be clearly understood as a nonlinear system.

In [38], the authors use the sum of the kinetic energy of the slider $\left(T_{s}\right)$ and the pendulum $\left(T_{b}\right)$ for a two-degree-offreedom rigid pendulum as shown in Fig. 13.

The differential equations of dynamics are derived from the sum of kinetic energy and the rigid pendulum's potential energy for both the first and second mutation. Also

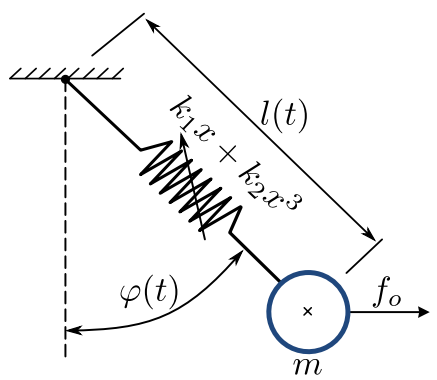

Fig. 12 Physical model for a nonlinear pendulum periodically excited for $f_{0}=Q_{0} \cos \omega t \sin \varphi$ 


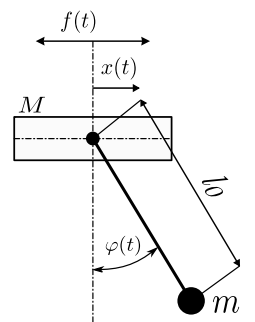

(a)

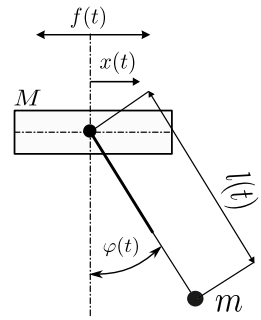

(b)

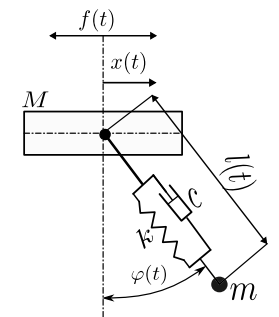

(c)
Fig. 13 The analyzed systems: rigid pendulum (a), the first (b) and the second (c) parametric mutation in an elastic form

considered the kinetic and potential energy for three degrees of freedom and derived the dynamics equation for both the first and second mutation. The second-order differential equations are given as follows:

$\begin{aligned} \ddot{l}(t)= & -\frac{c}{m} \dot{l}(t)-\frac{k}{m}\left(l(t)-l_{0}\right)+l(t) \dot{\varphi}(t)^{2} \\ & -\ddot{x}(t) \sin \varphi(t)+g \cos \varphi(t),\end{aligned}$

$\ddot{\varphi}(t)=-\frac{1}{l}(t)(2 \dot{\varphi}(t) \dot{l}(t)+\ddot{x}(t) \cos \varphi(t)+g \sin \varphi(t))$,

$\ddot{x}(t)=\frac{\left(c \dot{l}(t)+k\left(l(t)-l_{0}\right)\right) \sin \varphi(t)+Q_{0} \cos \omega t}{M}$.

where $l$-the pendulum elongation, $l_{0}$-constant length, $x$-the slider displacement, $\dot{\varphi}$ is the angular velocity, $\ddot{\varphi}$ is the angular acceleration, and $M$ is the mass of the slider body.

We see that a successive expansion in the forms of representation of the energies is introduced. The dynamic behavior is classified in two ways based on computational analysis of Lyapunov exponents using two proven methods. The numerically obtained phase planes and Poincaré maps show that some parameters exhibit similar dynamic behavior, such as quasi-periodic and chaotic motions.

A pendulum-spring system with variable-length is shown in Fig. 14 [39]. The equations of motion describe a threedegrees-of-freedom system, of which kinetic energy has the form:

$$
\begin{aligned}
& T(\varphi, l, x, \dot{\varphi}, \dot{l}, \dot{x})=\frac{1}{2}(M+m) \dot{x}^{2}(t)+\frac{1}{2} m\left[\dot{l}^{2}(t)\right. \\
& +l^{2}(t) \varphi^{2}(t)+2 \dot{x}(t) \dot{l}(t) \sin \varphi(t)+l(t) \dot{\varphi}(t) \sin \varphi(t) \\
& -\dot{l}(t) \cos \varphi(t))] .
\end{aligned}
$$

The potential energy has the form:

$$
\begin{aligned}
U(\varphi, \Delta l, x)= & \frac{1}{2} k\left(\Delta l(t)+\Delta l_{l t}\right)^{2}-m g(\Delta l(t) \\
& +l(t)) \cos \varphi(t) .
\end{aligned}
$$

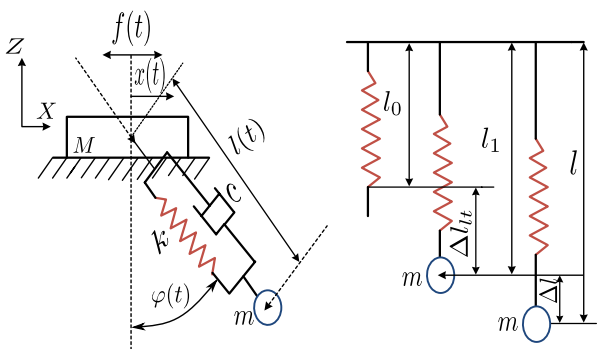

Fig. 14 Variable-length forced spring pendulum system of threedegrees-of-freedom (left), dimension of loaded and unloaded linear spring (right)

Applying the Euler-Lagrange equation and using the Rayleigh dissipation function of the pendulum, the equation of the pendulum angle, pendulum elongation, and the slider displacement were given respectively for the second derivative of state variables, i.e.:

$$
\begin{aligned}
\ddot{\varphi}(t)= & -\frac{1}{l(t)}(2 \ddot{\varphi}(t) \dot{l}(t)+p \cos \varphi(t)+g \sin \varphi(t)), \\
\ddot{l}(t)= & -\frac{c}{m} \dot{l}(t)-\frac{k}{m}\left(l(t)-l_{0}\right)+l(t) \dot{\varphi}^{2}(t) \\
& -\ddot{x}(t) \sin \varphi(t)+g \cos \varphi(t),
\end{aligned}
$$

where

$$
\begin{aligned}
p= & M^{-1}\left(c \dot{l}(t)+k\left(l(t)-l_{0}\right)\right) \sin \varphi(t) \\
& +Q_{0} M^{-1} \cos \omega t .
\end{aligned}
$$

The result shows that the variable-length spring pendulum suspended from the periodically forced slider can exhibit quasi-periodicity, and chaotic motions in a resonance state. It was concluded that near the resonance, the influence of coupling of bodies on the system dynamics could lead to unpredictable dynamical behavior.

In [40], a discrete wave modulated step function of length is used to excite a parametric pendulum subjected to a mathematical analysis similar in [39], Eqs. (43)-(45), and numerical modeling. Eq. (45) represent the acceleration of the slider. The numerical results show that semi-periodic solutions of ordinary differential equations with linear boundary value conditions exist. Conditions for synchronization of the systems and trajectories of the numerical solutions on time-history plots and phase planes confirm the analytical derivations and correctness of numerical modeling. 


\subsection{Applications}

After seeing the various analysis of the variable-length pendulum presented by different authors on different models, using different approaches, below are distinguish applications of the variable-length pendulum systems. There are various areas of application of these forms of pendulums, which include: energy harvesters (for example, in WEC), load-lifting devices (in cranes), robotics [4, 6, 41-47], and a pendulum-like robot in [29-31], to mention but a few.

In [41], the authors develop an experimental rig attached to a test device and obtain the required pendulum and energy harvester, see Fig. 15. A mathematical model is given in the form:

$$
\left\{\begin{array}{l}
I_{1} \ddot{\varphi}(t)+c \dot{\varphi}(t)+m \omega^{2} A l(t) \cos (\omega t) \sin \varphi(t)+ \\
m g l(t) \sin \varphi(t)=0, \\
I_{2} \ddot{\theta}(t)+c_{2} \dot{\theta}(t)+T_{f} \operatorname{sgn} \dot{\theta}(t)=0,
\end{array}\right.
$$

where $\theta$-angular position of flywheel, $A \cos \omega t$-kinematic vertical excitation, $T_{f}$-frictional torque.

Analyzing the dependence of the dynamical response on length of the pendulum, both numerically and experimentally, the shorter the pendulum length, the better gain of energy is achieved. It was concluded that several pendulums could be used to improve the dynamical response of the overall system.

In [43] present a flexural pendulum absorber that is attached to a linear system, as shown in Fig. 16. The system is modeled using a linear mass-spring-damper with mass $m$.

The following equations were used to describe the velocity $v$ at any point on the neutral beam axis.

$$
\begin{aligned}
v= & {\left[\dot{\bar{x}}_{e}(x, t)-\dot{\bar{y}}_{m}(t) \cos (\varphi(t))-\dot{\varphi}(t) \bar{y}_{e}(x, t)\right] i } \\
& +\left[\dot{\bar{y}}_{e}(x, t)+\dot{\bar{y}}_{m}(t) \sin (\varphi(t))+\dot{\varphi}(t) \bar{x}_{e}(x, t)+\dot{\varphi}(t)\right] j,
\end{aligned}
$$

where $y_{m}$-vertical displacement of the mass, $\bar{x}_{e}$-beam elastic displacement in $x$-direction, $\bar{y}_{e}$-beam elastic displacement in

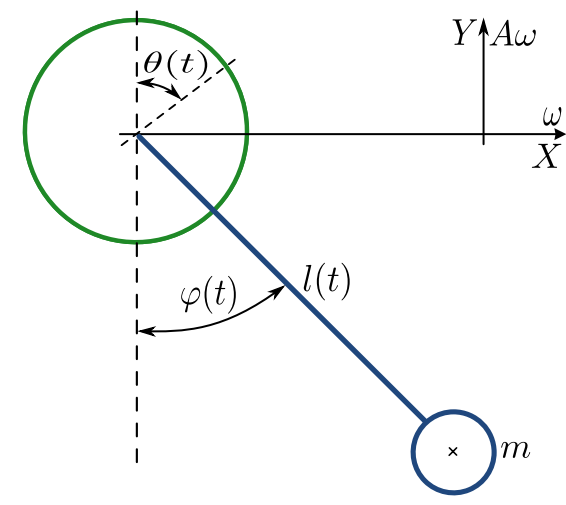

Fig. 15 Schematics of the system mathematical model the $y$-direction, $i, j$-component of vector. The equations of motion are described in term of $\bar{y}_{m}$ and $\varphi(t)$, and $\bar{x}_{e}$ and $\bar{y}_{e}$ for the primary and secondary equations, respectively.

The assumed flexural modes of the beam determined the possibility of Neimark-Sacke and Pitchfork's local bifurcation mode and resulted in a higher response rate for the system. The auto-parametric absorber rigid pendulum has shown to provide a longer lifespan than a standard rigid pendulum as provided by [36]. Therefore, auto-parametric pendulum absorption systems work well at a minimum amplitude of excitation.

In [6], a numerical simulation on the parametric equation of a pendulum and a real-time remote experimentation is conducted, as shown in Fig. 17.

The system kinetic and potential energies are considered, and using the Euler-Lagrange equation, the system equations are obtained:

$$
\begin{aligned}
& m_{1} l_{1}^{2}(t) \ddot{\varphi}_{1}(t)+m_{1} g l_{1}(t) \varphi_{1}(t)+k l_{3}^{2}(t)\left(\varphi_{1}(t)\right. \\
& \left.\quad-\varphi_{2}(t)\right)=0, \\
& m_{2} l_{2}^{2}(t) \ddot{\varphi}_{2}(t)+m_{2} g l_{2}(t) \varphi_{2}(t)+k l_{3}^{2}(t)\left(\varphi_{2}(t)\right. \\
& \left.\quad-\varphi_{1}(t)\right)=0,
\end{aligned}
$$

where $l_{1}$ and $l_{2}$ are the lengths varying parameters of the first and second pendulum, respectively, $l_{3}$ is the spring distance from the pivot.

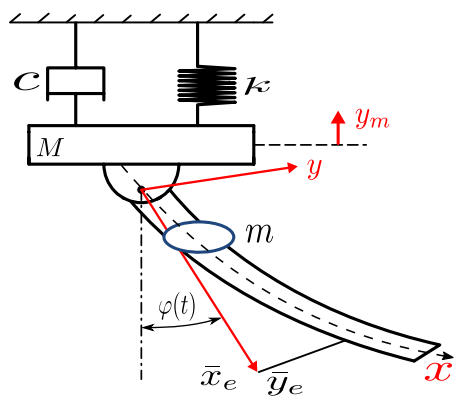

Fig. 16 Flexural pendulum absorber attached to a linear primary system

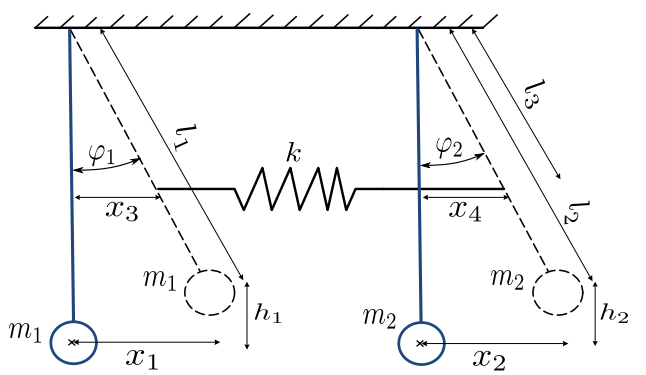

Fig. 17 Schematics of the connected pendulum system 


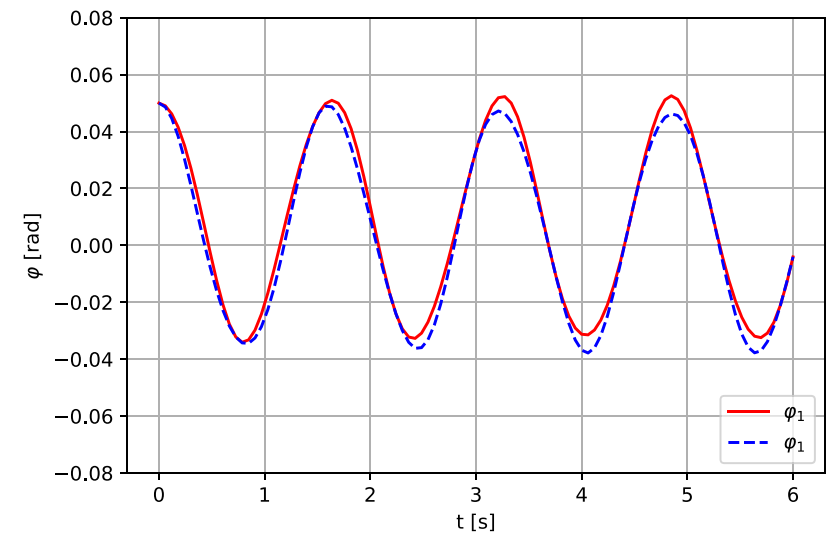

Fig. 18 Time domain plots for both pendulums in mode 1 with the parameters: $m_{1}=m_{2}=0.5 \mathrm{~kg}, l_{3}=0.55 \mathrm{~m}, \mathrm{~g}=9.8$, and $k=19.7 \mathrm{~N} / \mathrm{m}$

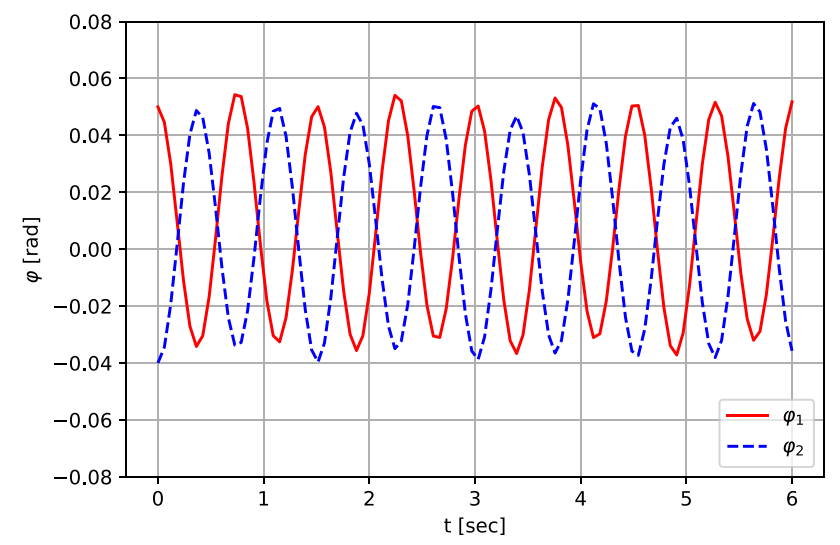

Fig. 19 Time domain plots for both pendulums in mode 2 with the parameters: $m_{1}=m_{2}=0.5 \mathrm{~kg}, l_{3}=0.55 \mathrm{~m}, g=9.8$, and $k=19.7 \mathrm{~N} / \mathrm{m}$

The simulation result shows a good agreement with the experimental data with an error at a low percentage. The dynamical model given by Eqs. (48) and (49) is solved numerically as shown in Figs. 18, 19 and 20. New research should investigate the effect of the change on the system's natural frequencies with significant changes in the sample systems such as spring and stiffness, bob size, etc.

Wave energy can be converted to other applicable forms of energy using the concept of the parametric pendulum. In [4], the concept of a parametric pendulum with a WEC was studied. It can be illustrated as in Figs. 21 and 22.

The equation of motion associated with the model in Fig. 21 follows:

$m l^{2}(t) \ddot{\varphi}(t)+c \dot{\varphi}(t)+m g l(t) \sin \varphi(t)=m l(t) \ddot{f}(t) \sin \varphi(t)$,

where $\ddot{f}(t)$ is the time-dependent excitation force.

The device can be actively controlled by the natural frequency of the pendulum and the amplitude of

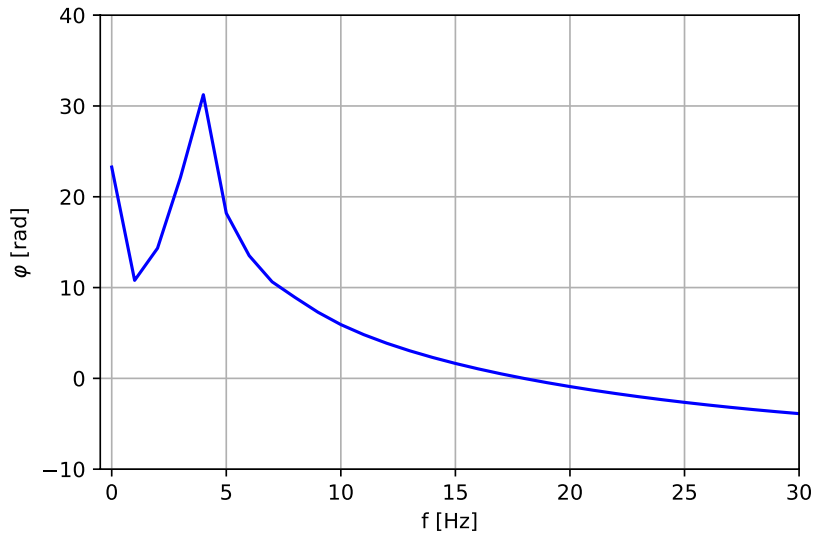

Fig. 20 Spectral response depicting combined modes of system vibration with the parameters: $m_{1}=m_{2}=0.5 \mathrm{~kg}, l_{3}=0.55 \mathrm{~m}, \mathrm{~g}=9.8$, and $k=19.7 \mathrm{~N} / \mathrm{m}$

Fig. 21 Original parametric pendulum in WEC concept
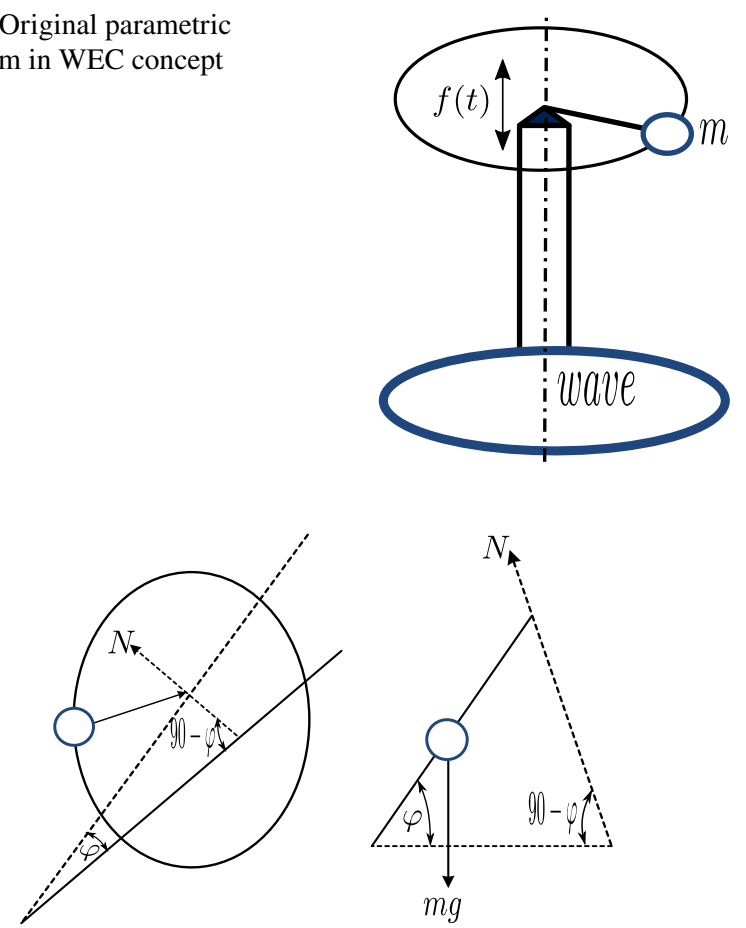

Fig. 22 Pendulum configuration with reduced gravity force with 3D (left) and side (right) view

excitation $A$ that can be lowered. For a harmonic forcing, $f(t)=A^{*} \cos (\omega t)$. Therefore, Eq. (50) can be rewritten:

$$
\begin{gathered}
m l^{2}(t) \ddot{\varphi}(t)+c \dot{\varphi}(t)+m g l(t) \sin \varphi(t) \\
\quad=-M l(t) \omega^{2} A^{*} \cos \omega t \sin \varphi(t),
\end{gathered}
$$

where $A^{*}$ is the amplitude along the $\operatorname{ramp} \frac{A}{\sin \varphi}, \varphi$ is the angle between the water surface and the pendulum's point of rotation. 
It was assumed that more energy could be collected by adding a flywheel to the design and thus, Eq. (51) becomes:

$$
\begin{aligned}
& m l^{2}(t)(1+\eta) \ddot{\varphi}(t)+c \dot{\varphi}(t)+m g l(t) \sin \beta \sin \varphi(t) \\
& \quad=m \ddot{l}(t) f(t) \sin \varphi(t),
\end{aligned}
$$

for $\eta=M R^{2} /\left(m l^{2}(t)\right)$.

The numerical and experimental results have confirmed the increase in the pendulum's rotational motion without any additional mechanisms. It has been found that new technology allows the pendulum to operate in response to a regular sea state without the use of new devices such as gearboxes or some other active control devices.

The work [44] is concerned with the primary parametric resonance as presented in Fig. 23. A triple pendulum (see Fig. 24) replaced the simple pendulum, estimated to make an angle of $120^{\circ}$ between the arms of the triple-pendulum with three equal masses distributed at the three arms.

For the standard design, the axis moves through $f(t)$, and the motion ratio is given as follows:

$$
\begin{aligned}
& \left(I_{c}+I_{v}\right) \ddot{\varphi}(t)+c \dot{\varphi}(t)+m\left(l_{1}(t)\right. \\
& \left.\quad-l_{2}(t)\right)[g+\ddot{f}(t)] \sin \varphi(t)=0,
\end{aligned}
$$

where $I_{c}$ is the constant of inertia of all the parts that are free to move in angular space, $I_{v}$ is the adjustable inertia, and $c$ is the viscous damping coefficient.

The natural frequency is given in Eq. (54) and is affected when the lengths $l_{1}$ and $l_{2}$ vary in time.

$\omega_{0}=\sqrt{m g \frac{\left(l_{1}(t)-l_{2}(t)\right)}{\left(I_{c}+I_{v}\right)}}$.

The moment of inertia is the varying $I_{v}$, which is altered by $l_{1}$ and $l_{2}$. The work aims to solve the problem of WEC application, and provides flexibility and configuration.

Logarithmic analysis techniques have been incorporated and are of great value. The value was used during the numerical simulation. The super harmonics and fast-moving design system was found to be;

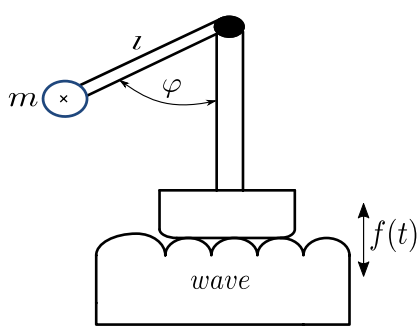

Fig. 23 Sketch of the pendulum-based WEC
Fig. 24 Sketch of the triple-pendulum that replaced the simple pendulum

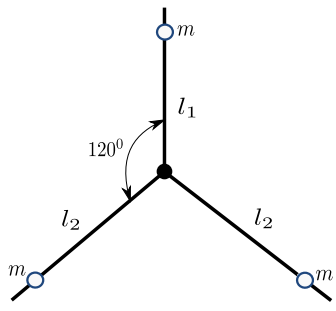

$\ddot{f}(t)=\omega^{2} r\left(\cos \omega t+\frac{\cos 2 \omega t}{j_{c}}\right)$,

where $r$-amplitude of excitation (crank-arm radius) and $j_{c}$ -ratio of the length of the crank.

Analysis of excitation were carried out with different values of $l$ and $v$, with varying configurations of $l_{1}$ and $l_{2}$. It was found that the triple-pendulum can change its size but does not control its response.

\subsection{Load Oscillations}

In [48], a water plant-related problem was used with dry friction, which added to the excitement of the water-filled container, see Fig. 25. Theory of Lyapunov stability is associated with the obtained time history of the charged water tank according to the level of the stick-slip phase and slip phase created by the Hopf bifurcation scenario of the equilibrium position.

Dynamics model of the system as shown in Fig. 25c is derived in the form:

$$
\begin{aligned}
(M+m) g \mu_{f}= & (M+m) \ddot{x}_{f} \\
& +\sum_{i=1}^{\infty} m_{i} l_{i} \ddot{\varphi}_{i}\left(\cos \varphi_{i}-\mu_{f} \sin \varphi_{i}\right) \\
& -\sum_{i=1}^{\infty} m_{i} l_{i} \dot{\varphi}_{i}^{2}\left(\sin \varphi_{i}+\mu_{f} \cos \varphi_{i}\right)+k x_{f},
\end{aligned}
$$

$m_{i} l_{i}^{2} \ddot{\varphi}_{i}+m_{i} l_{i} \ddot{x}_{f} \cos \varphi_{i}+m_{i} g l_{i} \sin \varphi_{i}+c_{i} \varphi_{i}=0$

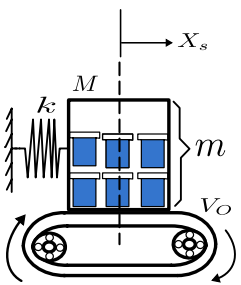

(a)

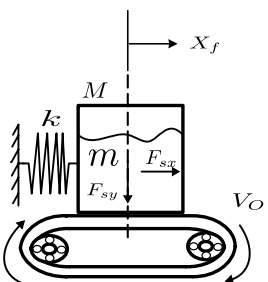

(b)

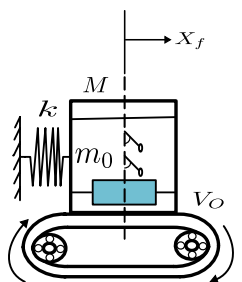

(c)
Fig. 25 Configurations of a tank on a moving belt: a solid-cargofilled tank (a); a liquid-filled tank (b); multidimensional-mode equivalent model of a liquid-filled tank (c) 
for $i=1,2, \ldots$.

Dynamical model for the system shown in Fig. 25a is derived in the form:

$$
\begin{aligned}
& (M+m) \ddot{x}_{s}+k\left[x_{s}+\frac{(M+m) g \mu_{0}}{k}\right]=(M+m) g \mu_{f}, \\
& (M+m) \ddot{x}_{s}+k\left[x_{s}+\frac{(M+m) g \mu_{0}}{g}\right]=F_{s x} \\
& \quad+\left(m_{g}+F_{s y}\right) \mu_{f},
\end{aligned}
$$

where $\mu_{f}$ is the coefficient of friction between the tank and belt, $\mu_{0}$ is expressed by $\mu_{f} v_{0}, v_{0}$ is the relative speed of the tank.

The stiffness of the spring, $k$ the roughness of the contact plates, and the ratio between the water and the reservoir contributed to the bifurcation area's change. Nonlinear vibrational phenomena can be studied and also more observations can be stored in the water-spring-water system placed on a moving belt.

In [49], the authors present a robust quasi-linear parameter varying tracking fault-tolerant control of a 3-DOF mechanical crane, see in Fig. 26. The control algorithm was created to minimize vibrations of load and to monitor the desired path using a quasi-linear parameter-varying system with $H_{\infty}$ basis.

The system's state-space model is presented in the equation:

$$
\begin{gathered}
\ddot{q}=-M(q)^{-1} c \dot{q}-M(q)^{-1} C(q, \dot{q}) \dot{q}-M(q)^{-1} \\
G(q)+M(q)^{-1} F
\end{gathered}
$$

where $M$ states the traveling and hoisting component of the crane. The $H_{\infty}$ performance guarantees robustness against noise measurement and faults.

The results show the efficacy of the proposed method. This is achieved by tracking a predefined position of the cart and load while oscillations are attenuated despite actuator faults. Noise measurement should be included. The slider mode controller can be used to compare the developed system with a complete nonlinear controller.

Fig. 26 Three degree of freedom mechanical crane

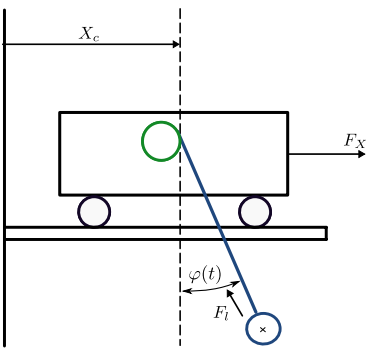

\section{A Variable-Length Pendulum system: Swinging Atwood's Machine}

One of the areas of the variable-length pendulum is the Swinging Atwood's Machine (SAM). In this case, the pendulum's mass swings in a two-dimensional plane, producing a chaotic behavior without colliding with the other mass known as the counterweight [50-52]. The inextensible massless string suspended on two frictionless pulleys connects the two groups (see also [53]), as presented in Fig. 27.

When the compensation is less than the pendulum mass, the system will have a large parameter space, which leads to rich dynamical behaviour $[50,54]$. These categories are terminating or non-terminating, chaotic or quasi-periodic, bounded or unbounded, singular or non-singular, which depends on the pendulum's reactive centrifugal force counteracting the counterweight [55-59].

\subsection{Equations of Motion}

The system is of two degrees of freedom. The equation of motion is derived using Lagrange equation $L=T-U$ and Hamiltonian $H=T+U$ [50], $T$ and $U$ are the kinetic and potential energies, respectively.

The two ordinary differential equations cover dynamics in $\varphi(t)$ and $l(t)$, since for the first state-space variable, we find

$\frac{\partial L}{\partial \varphi}=\frac{d}{d t}\left(\frac{\partial L}{\partial \dot{\varphi}}\right)$,

and for the second one, the following equation is applied

$\frac{\partial L}{\partial l}=\frac{d}{d t}\left(\frac{\partial L}{\partial \dot{l}}\right)$

Considering Fig. 27

$T=\frac{1}{2} M \dot{l}^{2}(t)+\frac{1}{2} m\left(\dot{l}^{2}(t)+l^{2}(t) \dot{\varphi}^{2}(t)\right)$,

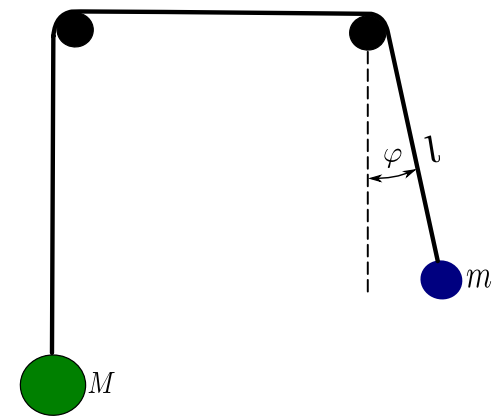

Fig. 27 The physical model of SAM 
$U=M g l(t)-m g l(t) \cos \varphi(t)$,

where $M$-the non-swinging mass, $m$-the swinging pendulum mass, $l(t)$-distance of the swinging mass to its pivot point.

We find the Lagrangian:

$L=\frac{1}{2} M \dot{l}^{2}(t)+\frac{1}{2} m\left(\dot{l}^{2}(t)+l^{2}(t) \dot{\varphi}^{2}(t)\right)$

$-\operatorname{Mgl}(t)+m g l(t) \cos \varphi(t)$.

Since the Hamiltonian is expressed in terms of the canonical momenta $p_{l}$ and $p_{\varphi}$ :

$H=\frac{p_{l}^{2}}{2(M+m)}+\frac{p_{\varphi}^{2}}{2 m l^{2}(t)}+M g l(t)-m g l \cos \varphi(t)$,

where $p_{l}=(M+m) \dot{l}(t), p_{\varphi}=m l^{2}(t) \dot{\varphi}(t)$.

The equations for the state-space variables $\varphi$ and $l$ are obtained from Eqs. (61) and (62), respectively:

$l(t) \ddot{\varphi}(t)+2 \dot{l}(t) \dot{\varphi}(t)+g \sin \varphi(t)=0$

$m l(t) \dot{\varphi}(t)^{2}-M g+m g \cos \varphi(t)=(M+m) \ddot{l}(t)$.

Taking the mass ratio $\mu_{m}=\frac{M}{m}$, Eq. (68) becomes

$\left(\mu_{m}+1\right) \ddot{l}(t)-l(t) \dot{\varphi}(t)^{2}+g\left(\mu_{m}-\cos \varphi(t)\right)=0$.

When the pulleys are considered, the system is said to have a moment of inertia $I_{p}$ and radius $R$, with effective mass $M_{t}$. The moment of inertia and the effective mass are given in Eqs. (70) and (71), respectively.

$I_{p}=R^{2}\left[\frac{M-m g(\Delta t)^{2}}{2 h}-(M-m)\right]$,

$M_{t}=M+m+\frac{2 I_{p}}{R^{2}}$.

The Hamiltonian is given as follows:

$H=\frac{1}{2}\left[\frac{p_{l}^{2}}{M_{t}}+\frac{\left(p_{\varphi}+R p_{l}\right)^{2}}{l^{2}(t)}\right]+g l(t)(M-$

$m \cos \varphi(t))+g R(m \sin \varphi(t)-M \varphi(t))$,

w h e r e $\quad p_{l}=-M_{t}(R \dot{\varphi}(t)-\dot{l}(t))$, a n d $p_{\varphi}=M_{t} R(R \dot{\varphi}(t)-\dot{l}(t))+m l^{2}(t) \dot{\varphi}(t)=-R p_{l}+m l^{2}(t) \dot{\varphi}(t)$.

The numerical solutions are presented in Figs. 28, 29, $30,31,32,33,34,35,36,37,38,39$. The value of $\varphi=\frac{\pi}{2}$ is kept constant with zero initial velocities in all cases, but the value of $\mu_{m}$ varies in time.

We observe rotations greater than $\pi$, but assuming that the string can wind up and unwind the string loop on the support, such angles are justified. The influence of the angle of contact of the string with the pulley has been omitted.

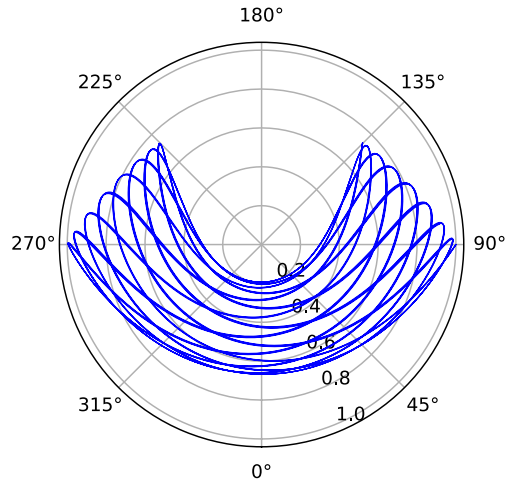

Fig. 28 An orbit of the SAM for $\mu_{m}=2.0, \varphi=\frac{\pi}{2}$, and initial velocity equal to zero. In the radial direction, we measure $l(t)$; in angular direction, we measure $\varphi(t)$

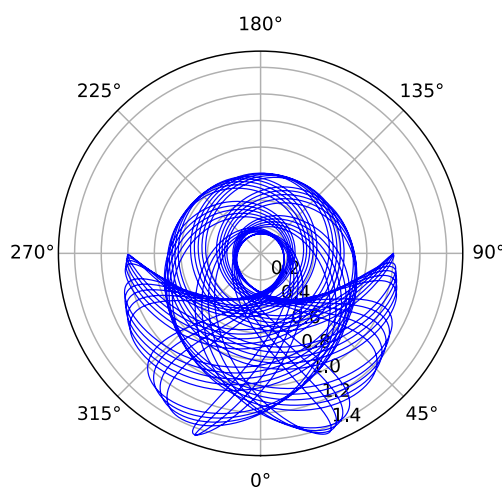

Fig. 29 An orbit of the SAM for $\mu_{m}=3.0, \varphi=\frac{\pi}{2}$, and initial velocity equal to zero. In the radial direction, we measure $l(t)$; in angular direction, we measure $\varphi(t)$

The Figs. 28, 29, 30, 31, 32, 33, 34, 35, 36, 37, 38, 39 show the SAM mass's nonsingular orbits under swinging, that is, no physical contact between the swinging assembly and the pulley. It can be seen that the SAM is integrable when $\mu_{m}=3$, and from the results, the system is not integrable for $\mu_{m} \in(0,1) \cup(3, \infty)$. The figure show the selection of nonsingular orbits of an orbit of SAM with different values of $\mu_{m}, \varphi=\frac{\pi}{2}$ and zero initial velocity.

\section{The Original Modification of SAM}

Based on the concepts delivered in the above review and to exhibit even potentially richer dynamics of the Swinging Atwood's Machine, an original and novel Modified SAM is proposed, as it can be seen in Fig. 40. Another spring pendulum is added on the opposite side of the non-swinging mass $M$. A suspension system with a stiffness $k$ and a damper $c$ placed between the two pendulums with masses $m_{1}$ and $m_{2}$. 


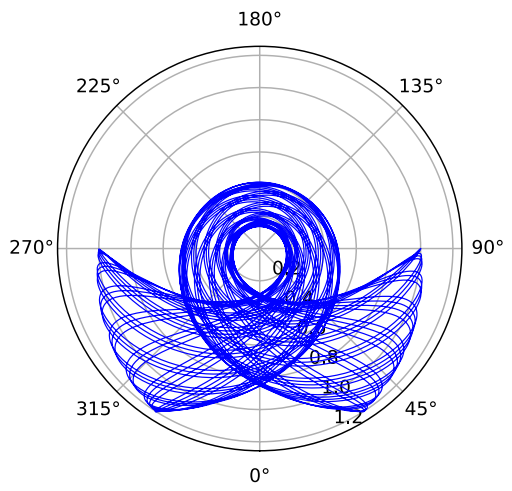

Fig. 30 An orbit of the SAM for $\mu_{m}=5.0, \varphi=\frac{\pi}{2}$, and initial velocity equal to zero. In the radial direction, we measure $l(t)$; in angular direction, we measure $\varphi(t)$

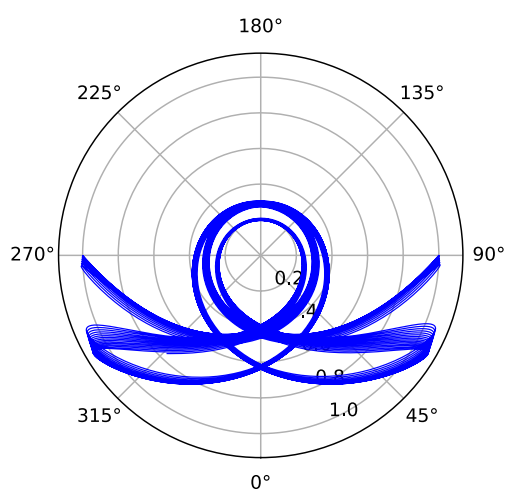

Fig. 31 An orbit of the SAM for $\mu_{m}=6.0, \varphi=\frac{\pi}{2}$, and initial velocity equal to zero. In the radial direction, we measure $l(t)$; in angular direction, we measure $\varphi(t)$

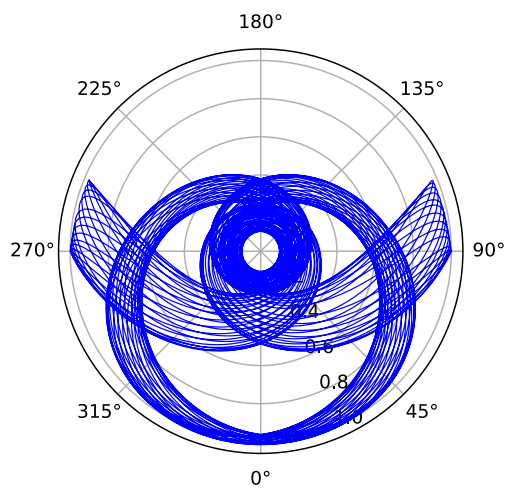

Fig. 32 An orbit of the SAM for $\mu_{m}=16.0, \varphi=\frac{\pi}{2}$, and initial velocity equal to zero. In the radial direction, we measure $l(t)$; in angular direction, we measure $\varphi(t)$

Point $0_{1}$ is fixed, while $0_{2}$ is movable and can oscillate in the plane $(X, Y)$, which allow the variation of the length $l_{1}$ and

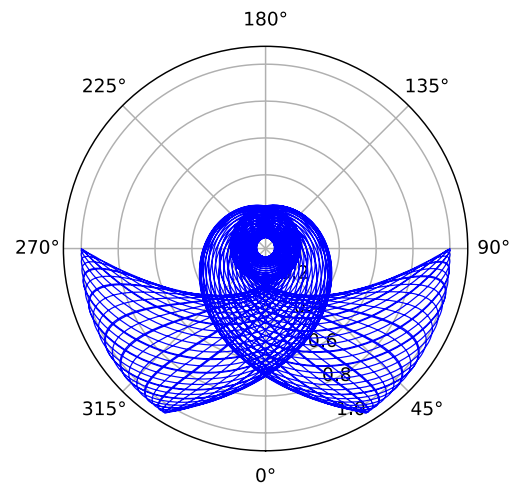

Fig. 33 An orbit of the SAM for $\mu_{m}=19.0, \varphi=\frac{\pi}{2}$, and initial velocity equal to zero. In the radial direction, we measure $l(t)$; in angular direction, we measure $\varphi(t)$

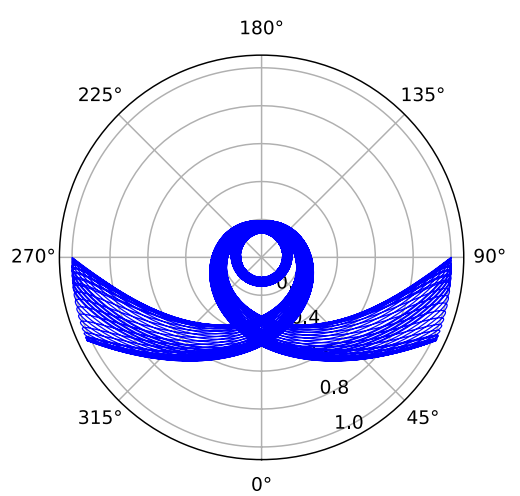

Fig. 34 An orbit of the SAM for $\mu_{m}=21.0, \varphi=\frac{\pi}{2}$, and initial velocity equal to zero. In the radial direction, we measure $l(t)$; in angular direction, we measure $\varphi(t)$

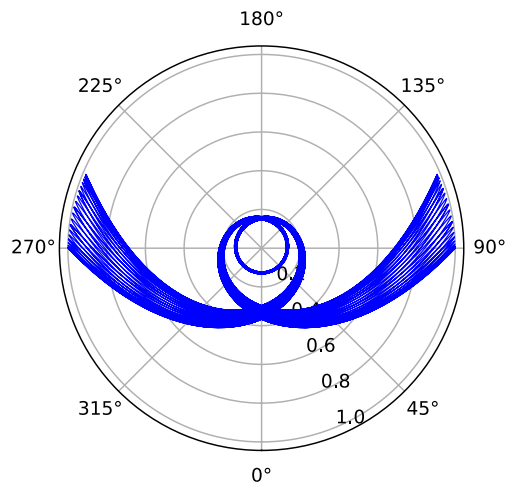

Fig. 35 An orbit of the SAM for $\mu_{m}=24.0, \varphi=\frac{\pi}{2}$, and initial velocity equal to zero. In the radial direction, we measure $l(t)$; in angular direction, we measure $\varphi(t)$

the double pendulum couplings. The length $l_{20}$ is measured between the two pendulums, and $l_{2}$ is the extension due to 


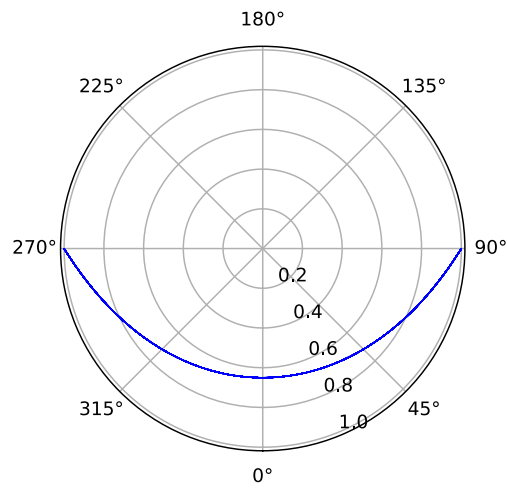

Fig. 36 A "Smile" orbit of the SAM for $\mu_{m}=1.7, \varphi=\frac{\pi}{2}$, and initial velocity equal to zero. In the radial direction, we measure $l(t)$; in angular direction, we measure $\varphi(t)$

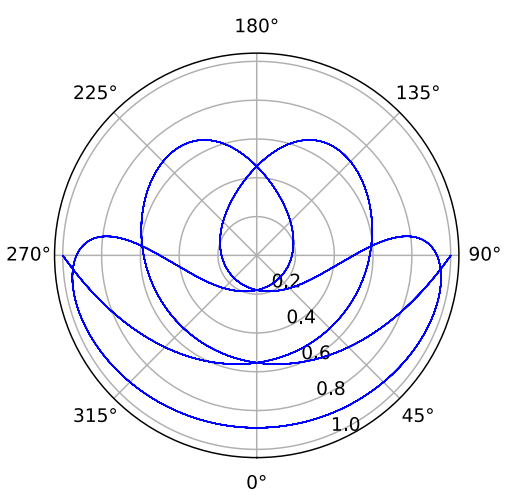

Fig. 37 An orbit of the SAM for $\mu_{m}=2.4, \varphi=\frac{\pi}{2}$, and initial velocity equal to zero. In the radial direction, we measure $l(t)$; in angular direction, we measure $\varphi(t)$

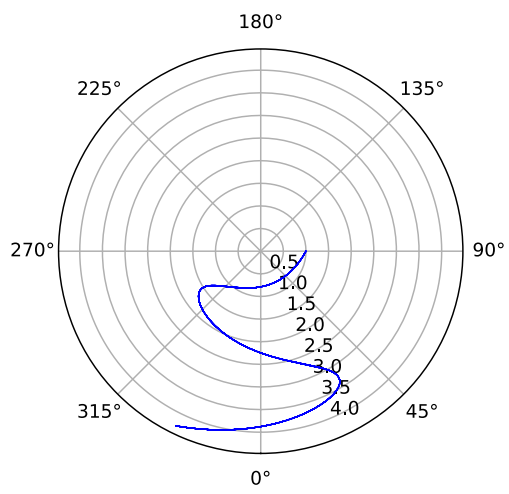

Fig. 38 An orbit of the SAM for $\mu_{m}=1.2, \varphi=\frac{\pi}{2}$, and initial velocity equal to zero. In the radial direction, we measure $l(t)$; in angular direction, we measure $\varphi(t)$

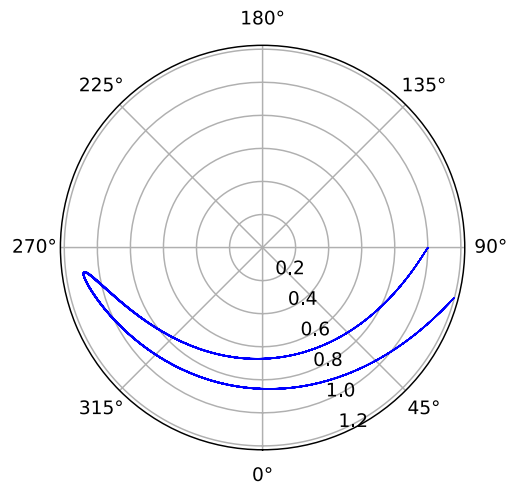

Fig. 39 An orbit of the SAM for $\mu_{m}=1.6, \varphi=\frac{\pi}{2}$, and initial velocity equal to zero. In the radial direction, we measure $l(t)$; in angular direction, we measure $\varphi(t)$

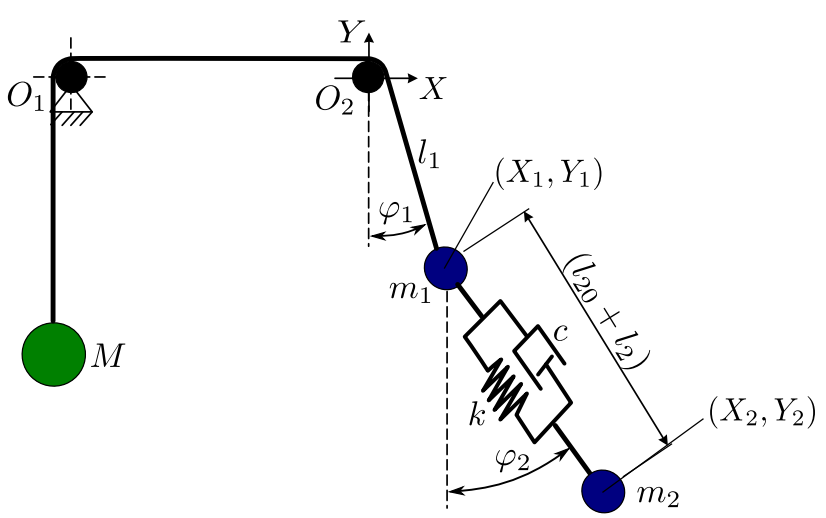

Fig. 40 Physical model of the proposed original Modified SAM

the spring between the two pendulums. Areas of application of the original Modified SAM model can include; load lifting devices such as cranes, wave variation, suspension systems, elastic robotic links, to mention a few. The Modified SAM also aimed at modeling physical systems and dynamics of loads carried by cranes placed on ships sailing at seas.

\subsection{The Equations of Motion of the Modified SAM}

Using the Euler-Lagrange method we find:

- the kinetic energy: For a simplicity we define $s_{21}(t)=\sin \left(\varphi_{2}(t)-\varphi_{1}(t)\right)$ and $c_{21}(t)=\cos \left(\varphi_{2}(t)-\varphi_{1}(t)\right)$. 


$$
\begin{aligned}
T= & \frac{1}{2} M \dot{l}_{1}^{2}(t)+\frac{1}{2} m_{1}\left(\dot{l}_{1}^{2}(t)+l_{1}^{2}(t) \dot{\varphi}_{1}^{2}(t)\right)- \\
& m_{2} \dot{l}_{1}(t) l_{2}(t) \dot{\varphi}_{2}(t) s_{21}(t)- \\
& l_{20} m_{2} \dot{l}_{1}(t) \dot{\varphi}_{2}(t) s_{21}(t)+ \\
& m_{2} l_{1}(t) \dot{l}_{2}(t) \dot{\varphi}_{1}(t) s_{21}(t)+ \\
& m_{2} l_{1}(t) l_{2}(t) \dot{\varphi}_{1}(t) \dot{\varphi}_{2}(t) c_{21}(t)+ \\
& l_{20} m_{2} l_{1}(t) \dot{\varphi}_{1}(t) \dot{\varphi}_{2}(t) c_{21}(t)+ \\
& m_{2} \dot{l}_{1}(t) \dot{l}_{2}(t) c_{21}(t)+ \\
& \frac{1}{2} m_{2} l_{2}^{2}(t) \dot{\varphi}_{2}^{2}(t)+l_{20} m_{2} l_{2}(t) \dot{\varphi}_{2}^{2}(t)+ \\
& \frac{1}{2} l_{20}^{2} m_{2} \dot{\varphi}_{2}^{2}(t)+\frac{1}{2} m_{2} l_{1}^{2}(t) \dot{\varphi}_{1}^{2}(t)+\frac{1}{2} m_{2} \dot{l}_{2}^{2}(t)+ \\
& \frac{1}{2} m_{2} \dot{l}_{1}^{2}(t) .
\end{aligned}
$$

- the potential energy:

$$
\begin{array}{r}
U=\frac{1}{2} k l_{2}^{2}(t)+M g l_{1}(t)-m_{1} g l_{1}(t) \cos \varphi_{1}(t)- \\
m_{2} g\left(l_{1}(t) \cos \varphi_{1}(t)+\left(l_{20}+l_{2}(t)\right) \cos \varphi_{2}(t)\right)
\end{array}
$$

where $M$-the non-swinging mass, $m_{1}$ and $m_{2}$ are the masses of the two pendulums, $\varphi_{1}$ and $\varphi_{2}$ are the angles between the movable pulley with length $l_{1}$ and the angle between the first pendulum with $\left(l_{20}+l_{2}\right)$, respectively, $l_{2}$ is the extension due to the spring with stiffness, $k$, and $c$ is the damper, $l_{1}$ is variable, since $l_{20}$ is constant, and $l_{2}$ is the elongation.

With the Lagrange equation, $L=T-U$ we find four degrees of freedom in the directions $l_{1}, l_{2}, \varphi_{1}$, and $\varphi_{2}$.

The Euler-Lagrange equation yields:

$\frac{d}{d t}\left(\frac{\partial L}{\partial \dot{q}_{i}}\right)-\frac{\partial L}{\partial q_{i}}+\frac{\partial R}{\partial \dot{q}_{i}}=0$,

where $i=1,2,3,4$, and $R$-Rayleigh dissipation function:

$$
\begin{aligned}
R\left(\dot{l}_{1}, \dot{l}_{2}, \dot{\varphi}_{1} \dot{\varphi}_{2}\right)= & \frac{1}{2} c\left[\frac{d\left(l_{20}+l_{2}(t)\right)}{d t}\right]^{2}= \\
& \frac{1}{c}\left[\frac{d l_{2}(t)}{d t}\right]^{2}=\frac{1}{2} c \dot{l}_{2}^{2}(t) .
\end{aligned}
$$

$c \dot{l}_{2}(t)$ states a non-conservative force.

There is constraint superposed on the first pendulum, i.e. if in the numerical integration procedure $l_{1}(t) \geq 0$ then we assume $l_{1}(t)=0$, since it has not been reported.

The following equations of motion are finally obtained:

- for $q_{1}=l_{1}$ :

$$
\begin{aligned}
& \ddot{l}_{1}(t)=\frac{-g m_{2} \sin \varphi_{2}(t) s_{21}(t)}{m_{1}}-\frac{g m_{2} \cos \varphi_{2}(t) c_{21}(t)}{m_{1}}+ \\
& \frac{c l_{2}(t) c_{21}(t)}{m_{1}}+\frac{k l_{2}(t) c_{21}(t)}{m_{1}}+l_{1}(t) \dot{\varphi}_{1}^{2}(t)+ \\
& \frac{g m_{2} \cos \varphi_{1}(t)}{m_{1}}+g \cos \varphi_{1}(t)-\frac{M g}{m_{1}} .
\end{aligned}
$$

- for $q_{2}=l_{2}$ :

$$
\begin{aligned}
& \ddot{l}_{2}(t)=\frac{g m_{2} \sin \varphi_{1}(t) s_{21}(t)}{m_{1}}+g \sin \varphi_{1}(t) s_{21}(t)- \\
& \frac{g m_{2} \cos \varphi_{1}(t) c_{21}(t)}{m_{1}}-g \cos \varphi_{1}(t) c_{21}(t)+ \\
& \frac{M g c_{21}(t)}{m_{1}}+l_{2}(t) \dot{\varphi}_{2}^{2}(t)+l_{20} \dot{\varphi}_{2}^{2}(t)+ \\
& \frac{g m_{2} \cos \varphi_{2}(t)}{m_{1}}+g \cos \varphi_{2}(t)-\frac{c \dot{l}_{2}(t)}{m_{2}}- \\
& \frac{c \dot{l}_{2}(t)}{m_{1}}-\frac{k l_{2}(t)}{m_{2}}-\frac{k l_{2}(t)}{m_{1}} .
\end{aligned}
$$

- for $q_{3}=\varphi_{1}$ :

$$
\begin{aligned}
& \ddot{\varphi}_{1}(t)=-\frac{g m_{2} \cos \varphi_{2}(t) s_{21}(t)}{m_{1} l_{1}(t)}+\frac{c \dot{l}_{2}(t) s_{21}(t)}{m_{1} l_{1}(t)}+ \\
& \frac{k l_{2}(t) s_{21}(t)}{m_{1} l_{1}(t)}+\frac{g m_{2} \sin \varphi_{2} c_{21}(t)}{m_{1} l_{1}(t)}-\frac{2 \dot{l}_{1} \dot{\varphi}_{1}}{l_{1}}- \\
& \frac{g m_{2} \sin \varphi_{1}}{m_{1} l_{1}(t)}-\frac{g \sin \varphi_{1}}{l_{1}(t)} .
\end{aligned}
$$

- for $q_{4}=\varphi_{2}$ :

$$
\begin{aligned}
\ddot{\varphi}_{2}(t)= & \frac{g m_{2} \cos \varphi_{1}(t) s_{21}(t)}{m_{1} l_{2}(t)+l_{20} m_{1}}+ \\
& \frac{g m_{1} \cos \varphi_{1}(t) s_{21}(t)}{m_{1} l_{2}(t)+l_{20} m_{1}}- \\
& \frac{M g s_{21}(t)}{m_{1} l_{2}(t)+l_{20} m_{1}}+\frac{g m_{2} \sin \varphi_{1}(t) c_{21}(t)}{m_{1} l_{2}(t)+l_{20} m_{1}}+ \\
& \frac{g m_{1} \sin \varphi_{1}(t) c_{21}(t)}{m_{1} l_{2}(t)+l_{20} m_{1}}-\frac{2 m_{1} l_{2} \dot{\varphi}_{2}}{m_{1} l_{2}(t)+l_{20} m_{1}}- \\
& \frac{g m_{2} \sin \varphi_{2}}{m_{1} l_{2}(t)+l_{20} m_{1}}-\frac{g m_{1} \sin \varphi_{2}}{m_{1} l_{2}(t)+l_{20} m_{1}} .
\end{aligned}
$$

\subsection{Simulation Results}

A simulation results of the described Modified SAM concept are presented in Figs. 41 and 42. The computation is performed in Python using the NumPy, SciPy, and Matplotlib libraries from Spyder in an Anaconda python environment. For simplicity, we assume that the initial angular positions of the pendulums are equal but in the opposite direction, 


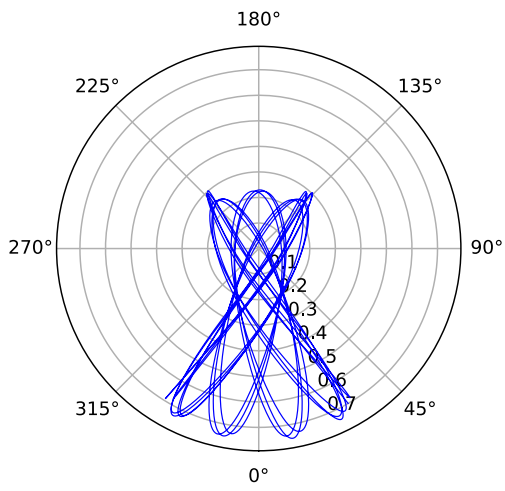

Fig. 41 An orbit of the Modified SAM for $M=15, m_{1}=3.5, \varphi_{1}=\frac{\pi}{6}$, and initial velocity equal to zero. In the radial direction, we measure $l_{1}(t)$; in angular direction, we measure $\varphi_{1}(t)$

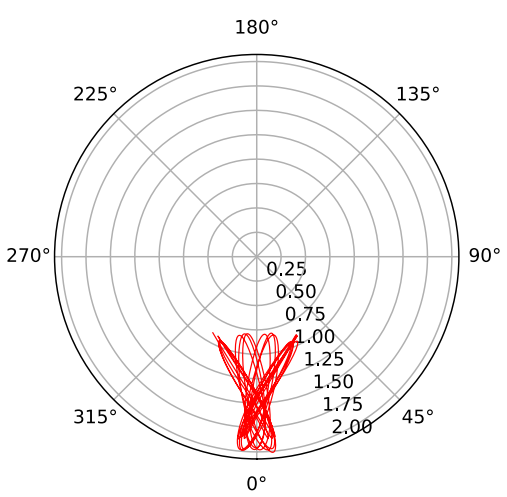

Fig. 42 An orbit of the Modified SAM for $M=15, m_{1}=3.5$, $m_{2}=3.7, \varphi_{1}=\frac{\pi}{6}, \varphi_{2}=-\frac{\pi}{6}$ and initial velocity equal to zero. In the radial direction, we measure $\left(l_{2} 0+l_{2}(t)\right)$; in angular direction, we measure $\varphi_{2}(t)$

i.e., $\varphi_{1}=-\varphi_{2}$. This application shows the Modified SAM's chaotic dynamics with a double pendulum and suspension system between the pendulums with different values $m_{1}, m_{2}$, $\varphi_{1}$ and $\varphi_{2}$.

Figure 41 shows the periodic motion of the first pendulum of mass $m_{1}$ with $\varphi_{1}$ and $l_{1}$, while Fig. 42 shows the periodic motion of the second pendulum of mass $m_{2}$ with $\varphi_{2}$ and $\left(l_{20}+l_{2}\right)$.

Current SAM concept is based on a variable-length double pendulum model with suspension between the two pendulums used. The presented results show nonsingular orbits after swinging-no physical contact between the swinging assembly and the fixed points. Interestingly, in some regimes, compact regions of attraction appear in the system, see Fig. 42.

\section{Conclusion}

The existing literature on variable-length/parametric pendulums is reviewed and particularly discussed. The mathematical model representing the system model and area of application in each of the references is presented. The method used, the finding, conclusions, and possible areas of improvement were clearly stated. It was suggested that the pendulum system could be applied in engineering applications. Also, consideration should be given to the pendulum length response to ensure that the response time is reduced to the minimum for adequate system handling.

Most of the literature does not consider how fast the length response changes in time. Only [32] tried to investigate the pendulum variable-length response time but had a negative linear trend and slope. The pendulum's variablelength response will be considered. It should ensure rapid changes in time.

A new model for the mechanical application of variable-length pendulum concept is proposed, the equations of dynamics are simulated numerically to prove richness of dynamical behaviour, as well as the obtained results are discussed.

The Modified SAM presents a novel SAM concept applicable in the modeling of engineering objects. It bases on a variable-length double pendulum with a suspension between the two pendulums. From the numerical simulation results, it is clearly seen that the nonlinear dynamics of the Modified SAM presented in current work can be thoroughly studied, and more modifications can be achieved. The new technique can reduce residual vibrations through damping when the desired level of an engineering object, e.g., a crane, is reached. It can also be used in the modeling of nonlinear mechatronic and robotic multidimensional systems. A deeper explanation and details on the string-pulley contact under variation of forces acting on the string will be developed in the future.

Acknowledgements This article has been completed while the first author Godiya Yakubu is the Doctoral Candidate in the Interdisciplinary Doctoral School at the Lodz University of Technology, Poland.

Funding This research was funded by Narodowe Centrum Nauki Grant No. 2019/35/B/ST8/00980 (NCN Poland).

\section{Declarations}

Conflict of interest The authors declare that they have no conflict of interest.

Open Access This article is licensed under a Creative Commons Attribution 4.0 International License, which permits use, sharing, adaptation, distribution and reproduction in any medium or format, as long as you give appropriate credit to the original author(s) and the source, provide a link to the Creative Commons licence, and indicate if changes 
were made. The images or other third party material in this article are included in the article's Creative Commons licence, unless indicated otherwise in a credit line to the material. If material is not included in the article's Creative Commons licence and your intended use is not permitted by statutory regulation or exceeds the permitted use, you will need to obtain permission directly from the copyright holder. To view a copy of this licence, visit http://creativecommons.org/licenses/by/4.0/.

\section{References}

1. Hatvani L (2015) On the parametrically excited pendulum equation with a step function coefficient. Int $\mathrm{J}$ Non Linear Mech 77:172-182. https://doi.org/10.1016/j.ijnonlinmec.2015.07.008

2. Chicone C (1999) Ordinary differential equations with applications, texts in applied mathematics, vol 34. Springer, Berlin

3. Xin X, Lin Y (2014) Control design and analysis for underactuated robotic systems. Springer, New York

4. Yurchenko D, Alevras P (2017) Parametric pendulum base wave energy converter. J Mech Syst Signal Process 99:504-515. https:// doi.org/10.1016/j.ymssp.2017.06.026

5. Yurchenko D, Alevras P (2013) Dynamics of the n-pendulum and its application to a wave energy converter concept. Int J Dyn Control 1:290-299. https://doi.org/10.1007/s40435-013-0033-x

6. Rajpal R, Kant M, Kuchibhatla SAR, Gangadharan KV (2018) Parametric modeling and real time remote experimentation of a reconfigurable coupled pendulum. Mater Today Proc. https://doi. org/10.1016/j.matpr.2018.10.210

7. Das A, Kumar K (2015) The dynamics of a parametrically driven damped pendulum. Int J Appl Mech Eng 20(2):257-266

8. Liang Z, Zhou Z (2017) Stable and unstable periodic solutions of the forced pendulum of variable length. Taiwanese J Math 21(4):791-806. https://doi.org/10.11650/tjm/7829

9. Reguera F, Dotti FE, Machado SP (2016) Rotation control of a parametrically excited pendulum by adjusting its length. Mech Res Commun 72:74-80. https://doi.org/10.1016/j.mechrescom. 2016.01.011

10. Xu X, Wiercigroch M, Cartmell MP (2005) Rotating orbits of a parametrically-excited pendulum. Chao Solitons Fractals 23(5):1537-1548. https://doi.org/10.1016/j.chaos.2004.06.053

11. Kapitaniak M, Czolczynski K, Perlikowski P, Stefanski A, Kapitaniak T (2014) Synchronous states of slowly rotating pendula. Phys Rep 541(1):1-44. https://doi.org/10.1016/j.physrep.2014.02.008

12. Czolczynski K, Perlikowski P, Stefanski A, Kapitaniak T (2012) Synchronization of slowly rotating pendulums. Int J Bifurc Chaos. https://doi.org/10.1142/S0218127412501283

13. Roura P, González JA (2010) Towards a more realistic description of swing pumping due to the exchange of angular momentum. IOP Publ 31(5):1195-1207. https://doi.org/10.1088/0143-0807/31/5/ 020

14. Olejnik P, Awrejcewicz J (2018) Coupled oscillators in identification of nonlinear damping of a real parametric pendulum. J Mech Syst Signal Process 98:91-107

15. Akulenko LD, Nesterov SV (2018) Parametric oscillations of the kochin oscillator with dissipation. Doklady Phys 63:128-131. https://doi.org/10.1134/S1028335818030084

16. Strinati MC, Aharonovich I, Ben-Ami S, Torre EGD, Bello L, Pe'er A (2020) Coherent dynamics in frustrated coupled parametric oscillators. New J Phys. https://doi.org/10.1088/1367-2630/ aba573

17. Luo ACJL, Ma H (2017) Bifurcation trees of periodic motions to chaos in a parametric duffing oscillator. Int J Dyn Control 6(2):425-458. https://doi.org/10.1007/s40435-017-0314-x
18. Jing Z, Yang $\mathbf{J}$ (2006) Complex dynamics in pendulum equation with parametric and external excitations i. Int J Bifurc Chaos 16(10):2887-2902. https://doi.org/10.1142/S0218127406016525

19. Li C, Zhang Z, Liu X, Shen Z (2019) An improved principle of rapid oscillation suppression of a pendulum by a controllable moving mass: theory and simulation. Shock Vib 2019:1-11. https://doi.org/10.1155/2019/5346463

20. Fan J, Liu T, Chen S (2019) Analysis of dynamical behaviors of a 2-dof friction-induced oscillator with one-sided impact on a conveyor belt. Nonlinear Dyn 97:797-830. https://doi.org/10. 1007/s11071-019-05014-5

21. Gao M, Fan J (2020) Analysis of dynamical behaviors of a 2-dof friction oscillator with elastic impacts and negative feedbacks. Nonlinear Dyn 102(1):45-78. https://doi.org/10.1007/ s11071-020-05904-z

22. Malits P (2010) Relations between Mathieu functions of the first and second kind. Integral Trans Spec Funct 21(6):423-436. https://doi.org/10.1080/10652460903360499

23. Magnus S, Winkler W (2013) Hill's equation. Courier Publishing, London

24. Richards JA (1983) Analysis of periodically time-varying systems. Springer, Berlin

25. Seyranian AP, Seǐranyan AA (2013) Breakup of resonance zones for Meissner is equation with small damping. Vestnik Moskov Univ Ser Mat Mekh 5:53-59

26. Wright JA, Bartuccelli M, Gentile G (2017) Comparisons between the pendulum with varying length and the pendulum with oscillating support. J Math Anal Appl 449(2):1684-1707. https://doi. org/10.1016/j.jmaa.2016.12.076

27. Huff A, Thompson J, Pate J, Chiao R, Sharping JE (2019) A parametric oscillator for class room demonstration or student laboratory. Eur J Phys 40(6):1-12. https://doi.org/10.1088/1361-6404/ ab2fe9

28. Kuře M, Bušek J, Vyhlídal T, Niculescu S-I (2021) Algorithms for cable-suspended payload sway damping by vertical motion of the pivot base. Mech Syst Signal Process 149:107-131. https:// doi.org/10.1016/j.ymssp.2020.107131

29. Zelei A, Bencsik L, Kovács LL, Stépán G (2012) Redundancy resolution of the underactuated manipulator acroboter, in RoManSy 19. In: CISM-IFTOMM symposium on robot design dynamics and control, Paris, France June 12-15, pp 233-240. https://doi. org/10.1007/978-3-7091-1379-0_29

30. Zelei A, Kovács LL, Stépán G (2011) Computed torque control of an under-actuated service robot platform modeled by natural coordinates. Commun Nonlinear Sci Numer Simul 16(5):2205-2217. https://doi.org/10.1016/j.cnsns.2010.04.060

31. Zana RR, Zelei A (2021) Feedback motion control of a spatial double pendulum manipulator relying on swept laser based pose estimation. Int J Optomechatron 15(1):32-60. https://doi.org/10. 1080/15599612.2021.1890284

32. Caccamoa MT, Magazú S (2018) Variable length pendulum analyzed by a comparative fourier and wavelet approach. Revista Mexicana de Física 64:81-86

33. Ftorek B, Oršanský P, Šamajová H (2018) Parametric oscillations of the mechanical systems. MATEC Web of Conf 157:1-7. https:// doi.org/10.1051/matecconf/201815708002

34. Kovaleva M, Manevitch L, Romeo F (2019) Stationary and nonstationary oscillatory dynamics of the parametric pendulum. Commun Nonlinear Sci Numer Simul 76:1-11. https://doi.org/ 10.1016/j.cnsns.2019.02.016

35. Roberto DS, Pradoa SD, Fernandes HA (2017) A new look on the stabilization of inverted pendulum with parametric excitation and large random frequencies: analytical and numerical approaches. Commun Nonlinear Sci Numer Simul 51:105-114. https://doi.org/ 10.1016/j.cnsns.2017.04.002 
36. Krasilnikov P, Gurina T, Svetlova V (2018) Bifurcation study of a chaotic model variable-length pendulum on a vibrating base. Int J Non Linear Mech 105:88-98. https://doi.org/10.1016/j.ijnon linmec.2018.06.011

37. Luo ACJ, Yuan Y, Caramel S (2020) Bifurcation trees of period-1 to period-2 motions in a periodically excited nonlinear spring pendulum. J Vib Test Syst Dyn 4:201-248

38. Śmiechowicz W, Loup T, Olejnik P (2019) Lyapunov exponents of early stage dynamics of parametric mutations of a rigid pendulum with harmonic excitation. Math Comput Appl 24(4) https://www. mdpi.com/2297-8747/24/4/90

39. Pietrzak P, Ogińska M, Krasuski T, Figueiredo K, Olejnik P (2018) Near the resonance behavior of a periodically forced partially dissipative three-degrees-of-freedom mechanical system. Latin Am J Sci Struct. https://doi.org/10.1590/167978254423

40. Olejnik P, Fečkan M, Awrejcewicz J (2018) Analytical and numerical study on a parametric pendulum with the step-wave modulation of length and forcing. Int J Struct Stab Dyn. https://doi.org/ $10.1142 / \mathrm{S} 0219455419410062$

41. Marszal M, Witkowski B, Jankowski K, Perlikowski P, Kapitaniak $\mathrm{T}$ (2017) Energy harvesting from pendulum oscillations. Int J Non Linear Mech 94:251-256. https://doi.org/10.1016/j.ijnonlinmec. 2017.03.022

42. Bek MA, Amer TS, Almahalawy A, Elameer AS (2021) The asymptotic analysis for the motion of 3dof dynamical system close to resonances. Alexandria Eng J 60(4):3539-3551

43. Mahmoudkhani S (2018) Improving the performance of autoparametric pendulum absorbers by means of a flexural beam. J Sound Vib 425:103-123. https://doi.org/10.1016/j.jsv.2018.03. 025

44. Alevras P, Brown I, Yurchenko D (2015) Experimental investigation of a rotating parametric pendulum. Nonlinear Dyn 81:201213. https://doi.org/10.1007/s11071-015-1982-8

45. Starosta R, Sypniewska-Kamińska G, Awrejcewicz J (2017) Quantifying non-linear dynamics of mass-springs in series oscillators via asymptotic approach. Mech Syst Signal Process 89:149_ 158. https://doi.org/10.1016/j.ymssp.2016.07.022

46. Selyutskiy YD, Holub APH, Dosaev MZ (2019) Elastically mounted double aerodynamic pendulum. Int J Struct Stab Dyn. https://doi.org/10.1142/S0219455419410074
47. Wang Y-J, Chen C-D, Lin C-C, Yu J-H (2015) A nonlinear suspended energy harvester for a tire pressure monitoring system. Micromachines 6(3):312-327

48. Li J, Cao D, Pan K (2020) Dry-friction-induced self-excitation of a rectangular liquid-filled tank. Nonlinear Dyn. 102:1337-1359. https://doi.org/10.1007/s11071-020-05971-2

49. López-Estrada FR, Santos-Estudillo O, Valencia-Palomo G, Gómez-Peñate S, Hernándex-Gutiérrez C (2020) Robust qlpv tracking fault-tolerant control of a 3 dof mechanical crane. Math Comput Appl 25(3):1-12. https://doi.org/10.3390/mca25030048

50. Elmandouh AA (2016) On the integrability of the motion of 3d-swinging Atwood machine and related problems. Phys Lett A 380(9):989-991

51. Prokopenya AN (2017) Motion of a swinging Atwood's machine: simulation and analysis with mathematica. Math Comput Sci 11:417-425. https://doi.org/10.1007/s11786-017-0301-9

52. Tufillaro NB, Abbott TA, Griffiths DJ (1984) Swinging Atwood's machine. Am J Phys 52(895):895-903. https://doi.org/10.1119/1. 13791

53. Tufillaro NB (1994) Teardrop and heart orbits of a swinging Atwood's machine. Am J Phys 62(3):231-233

54. Tufillaro NB, Nunes A, Casasayas J (1998) Unbounded orbits of a swinging Atwood's machine. Am J Phys 56:1117-1120

55. Moreira IC, Almeida MA (1991) Noether symmetries and the swinging Atwood machine. J Phys II 1(7):711-715

56. Casasayas J, Nunes A, Tufillaro NB (1990) Swinging Atwood's machine: integrability and dynamics. J Phys II 51:1693-1702

57. Yehia HM (2006) On the integrability of the motion of a heavy particle on a tilted cone and the swinging Atwood's machine. Mech Res Commun 33(5):711-716. https://doi.org/10.1016/j. mechrescom.2005.06.015

58. Nunes A, Casasayas J, Tufillaro N (1995) Periodic orbits of the integrable swinging Atwood's machine. Am J Phys 63(2):121126. https://doi.org/10.1119/1.17967

59. Pujol O, Pérez JP, Ramis JP, Simó C, Simon S, Weil J (2010) Swinging Atwood machine: experimental and numerical results, and a theoretical study. Phys D Nonlinear Phenomena 239(12):1067-1081. https://doi.org/10.1016/j.physd.2010.02.017

Publisher's Note Springer Nature remains neutral with regard to jurisdictional claims in published maps and institutional affiliations. 